Submitted to ApJ

\title{
Cassini States with Dissipation: Why Obliquity Tides Cannot Inflate Hot Jupiters
}

\author{
Daniel C. Fabrycky, Eric T. Johnson, and Jeremy Goodman \\ Department of Astrophysical Sciences, Princeton University, Princeton, NJ 08544
}

\begin{abstract}
Some short-period exoplanets ("hot Jupiters") are observed by their transits to have anomalously large radii. It has been suggested that these planets are in a resonance involving persistent misalignment and synchronous precession of their spin and orbital angular momenta - a Cassini state - and that the attendant tidal heating inflates the planet. We argue against this. Using explicit tidal integrations, we show that although an oblique Cassini state can dissipate many times the planet's rotational energy, the rate of dissipation must be much less than hypothesized. Dissipation causes the planetary spin to lie at an angle to the plane containing the orbital and total angular momenta. If dissipation is too rapid, this angle becomes so large that Cassini equilibrium is lost. A separate consideration limits the total energy that can be extracted from the orbit. The source of the torque on the orbit, either an oblique parent star or an inclined third body, aligns with the orbit as it absorbs the angular momentum shed by the planet. Alignment removes the orbital precession required by the Cassini state. In combination with observational bounds on the mass and semimajor axis of a possible second planet and with bounds on the stellar rotation and obliquity, these constraints make it very unlikely that obliquity tides can be the explanation for inflated hot Jupiters, especially HD 209458b.
\end{abstract}

Subject headings: celestial mechanics, planetary systems, stars: individual (HD 209458), methods: analytical, methods: numerical

\section{Introduction}

Transit observations have exposed the limits of our understanding of the structure of short period extrasolar giant planets, or hot Jupiters. The models of transiting planet HD 209458b constructed by Bodenheimer, Lin. \& Mardling (2001) gave the first indication of a discrepancy between the theoretically predicted and observed radii of the largest hot Jupiters. While standard evolutionary models for irradiated giant planets faithfully represent the majority of the 14 known transit cases, the conclusion persists that the models underpredict the radii of several hot Jupiters 
by 10 - 20\% (Burrows et al. 2006a; Arras \& Bildsten 2006; Fortnev et al. 2006a; Guillot 2005). At the large end of the size distribution, planets HD 209458b (Knutson et al. 2007), WASP-1b (Shporer et al. 2006), and HAT-P-1b (Bakos et al. 2006) present the most serious challenge to expected rates of contraction. Resolution of the apparent radius problem will require simultaneous treatment of several outstanding issues, with competing effects. First, the problem is ameliorated somewhat by distinguishing the photospheric radius from the "transit radius," which is the impact parameter of photons that travel a chord of unit optical depth through the planetary atmosphere. The latter radius is larger by as much as $\sim 5 \%$ for planets with low surface gravity (Baraffe et al. 2003; Burrows et al. 2003, 2006a). Secondly, significant enlargement can be achieved in two ways; either by tapping an additional heat source (Bodenheimer et al. 2001) or by insulating the interior with a supersolar abundance of metals in the atmosphere, thereby retarding its contraction (Burrows et al. 2006a). Conversely, more rapid contraction may result if global redistribution of the insolation is inefficient, allowing an increased cooling flux to escape via the planet's cold night side (Guillot \& Showman 2002). Finally, planet radii are greatly reduced by the inclusion of heavy elements in the interior, whether dispersed or as a solid core, for which a mass $10-40$ times the mass of the Earth may be typical (Bodenheimer et al. 2003; Fortney et al. 2006a; Burrows et al. 2006a).

Burrows et al. (2006a) argue that, to within the observational uncertainties in radius and age, with no additional heating mechanism, the radii of all transiting hot Jupiters are consistent with evolutionary models if supersolar atmospheric metallicities are allowed. The authors rely upon heterogeneity in both atmospheric abundances and core mass. In particular, Burrows et al. fit coreless models with 10 times solar atmospheric abundances to WASP-1b (within the $1 \sigma$ error bar), HAT-P-1b (within $1 \sigma$ ), and HD 209458b (within $2 \sigma$ ). A concern is that the inflated radii of these models require that the metals be sequestered in the atmosphere; supersolar abundances throughout the planet might be expected to result in a net contraction (Fortney et al. 2006b). Observations of thermal emission from hot Jupiters also bear on the relevance of such models. Broadband detections of infrared emission at secondary eclipse have been reported for HD 209458b (Deming et al. 2005), TrES-1 (Charbonneau et al. 2005), and HD 189733b (Deming et al. 2006). Corresponding theoretical spectra were constructed by Seager et al. (2005), Barman et al. (2005), Fortney et al. (2005), and Burrows et al. (2005, 2006b). All obtained fits to the emission measurements while assuming solar abundances. None predicted metallicity as large as required by Burrows et al. (2006a) for HD 209458b, although Fortney et al. (2005) found marginally improved fits for TrES-1 using $3-5$ times solar abundances. Interpretation should nonetheless be cautious, as the continua of the model spectra depend more strongly on the degree of horizontal heat transport than on metallicity. In fact there is no consensus among these works as to whether the data indicate that the reradiation of absorbed stellar flux occurs locally, is globally uniform, or is intermediate between these limits. This points to another critical issue; insofar as the photospheric temperature is an indicator of the temperature near the base of the radiative zone, the observed day-night temperature contrast can constrain the redistribution of heat at depth. The contraction rates computed by Burrows et al. hinge on thorough redistribution and isothermal conditions along 
the radiative-convective boundary, where the cooling flux is determined (Arras \& Bildsten 2006). Such an isotherm bounding the convective interior was seen in the numerical simulations of global circulation by Iro et al. (2005). However, if this deep isotherm does not exist, then additional heating is indeed required to offset the steeper temperature gradient on the night side. Harrington et al. (2006) reported measurements of the phase curve of (non-transiting) hot Jupiter $v$ Andromedae b that imply a day-night contrast perhaps exceeding $10^{3} \mathrm{~K}$. However, from simulations with a deep isotherm, Iro et al. predicted contrasts not larger than $600 \mathrm{~K}$ for HD $209458 \mathrm{~b}$ (despite the fact that HD 209458b receives greater stellar flux than $v$ And b). As a final point, the first spectroscopic measurements of thermal emission have come with their own surprises. The mid-infrared spectra of HD 189733b (Grillmair et al. 2007) and HD 209458b (Richardson et al. 2007; Swain et al. 2007) are consistent with a Rayleigh-Jeans continuum, lacking the anticipated absorption bands blueward of $9 \mu \mathrm{m}$ associated with water and methane. These observations of hot Jupiter atmospheres will need to be understood theoretically before a conclusion can be reached regarding heat retention. A continual heating mechanism may yet play a principal role.

Augmentation of a Jovian planet's intrinsic luminosity causes an adjustment to a new hydrostatic equilibrium with an expanded radius. The estimated heating rates necessary to account for the inflated hot Jupiters are in the range $10^{26}-10^{28} \mathrm{erg} \mathrm{s}^{-1}$, which is $\lesssim 5 \%$ of the intercepted stellar luminosity and is a few orders of magnitude larger than the intrinsic cooling luminosity (Burrows et al. 2006a; Bodenheimer et al. 2003, 2001; Baraffe et al. 2003). This energy may be drawn from either the stellar insolation or the orbit. Guillot \& Showman (2002) pointed out that one-sided irradiation should drive an atmospheric heat engine that converts the insolation into bulk flow kinetic energy. Inflation would require that a fraction of the kinetic energy be dissipated at depths where the radiative timescale is sufficiently long. Numerical global circulation models that address this mechanism have yet to converge on several key factors, including characteristic wind velocities and global flow patterns. While Showman \& Guillot (2002) found substantial downward transport of kinetic energy in simulation, Burkert et al. (2005) found little vertical transport and near complete dissipation at shallow depths. A further puzzle is that Harrington et al. (2006) observed the phase curve of $v$ And $\mathrm{b}$ to be consistent with an $11^{\circ}$ lag between full phase and the substellar point. A superrotational wind like that predicted by Showman \& Guillot (2002) and Cooper \& Showman (2005) for HD 209458b, with a $60^{\circ}$ advance, is strongly disfavored by the data. Presuming that dissipation does occur at sufficient depth to affect the radius, one has to explain why this mechanism - or any other that appeals to characterstics common to all hot Jupiters - does not inflate all currently observed transiting planets. A possible answer involves competition among conflicting factors: for example, auxiliary heating offset by variable core masses.

An alternative is that tidal forces may heat the planet at the expense of the orbit. Tidal dissipation naturally depends upon variable orbital and perhaps other parameters. The action of tides is to align the spin and orbital angular momenta, synchronize the rotational and orbital periods, and circularize the orbit. The efficiency with which hot Jupiters are thought to dissipate tidal oscillations implies that alignment and synchronization occur on $10^{5}-10^{6} \mathrm{yr}$ timescales and 
circularization occurs on $10^{8}-10^{9} \mathrm{yr}$ timescales. The eccentricity and/or obliquity must be excited continually in order for tides to persist for several Gyr. Unlike the case of insolation-driven heating, ongoing tidal heating requires special orbital configurations, potentially involving a third body. Bodenheimer et al. (2001) and Bodenheimer et al. (2003) have suggested that eccentricity could be forced by a companion planet, and they have estimated that a sustained eccentricity $e \gtrsim 0.03$ would be sufficient to explain the radius of HD 209458b. Recent radial velocity measurements (Laughlin et al. 2005a) and timings of secondary eclipse (Deming et al. 2005) have tightened the constraints on the eccentricity of this system, but because of degeneracy with the longitude of periastron, cannot yet rule out $e=0.03$ with $95 \%$ confidence. An innovative possibility, put forth by Winn \& Holman (2005), is that a planet could occupy a so-called Cassini state in which its obliquity, rather than its eccentricity, is forced.

G. D. Cassini published three empirical laws in 1693 describing the spin state of the moon. Cassini's laws state that (1) the moon's rotational and orbital periods are synchronous, (2) its equatorial plane maintains constant inclination to the plane of the ecliptic, and (3) its spin axis remains coplanar with the normal to its orbit and the normal to the ecliptic. The first law is an outcome of tidal evolution for bodies with a permanent quadrupolar distortion, like the moon, or for fluid bodies in circular, stationary orbits (Murray \& Dermott 1999). The second and third laws characterize a Cassini state, which is an equilibrium for the motion of the spin axis of a body in a uniformly precessing Keplerian orbit. Up to four Cassini states may exist for a given system, each with a preferred obliquity. The moon occupies Cassini state 2 (see numbering scheme in \$2) with obliquity $\theta \approx 6.7$. Resonance between the precession frequency of the orbit and the natural precession frequency of the spin determines the values of the Cassini obliquities. The obliquity of a dissipative body is driven toward a Cassini state, which is a quasi-equilibrium in the sense that the body continues to experience an obliquity tide so long as $\theta$ is nonzero. In this paper we determine the effect of the attendant obliquity tide on the evolution and survival of Cassini states for hot Jupiters.

Levrard et al. (2007) have recently argued that the probability of a planet becoming trapped in an oblique Cassini state is rather small. Such probabilities depend upon the early evolution of the star and perhaps of its protostellar disk. Independently of initial conditions, however, there are reasons to doubt whether a hot Jupiter can remain in Cassini state 2 while experiencing ongoing tidal heating sufficient to affect the planet's radius measurably. Levrard et al. (2007) themselves have pointed out that maintenance of the oblique Cassini states requires a balance between the torque associated with dissipation in the planet and a nondissipative torque on the orbit due either to an oblate and oblique stellar primary or to a third body. Since the nondissipative torque on the orbit is likely to be small, we argue that the Cassini state can be maintained only by assuming a dissipation rate in the planet that is too small to inflate its radius significantly. Also, Levrard et al. (2007) considered only the balance of torques tending to alter the obliquity. Using a specific tidal model, we show that a stronger constraint results from requiring the spin to equilibrate in the azimuthal direction. 
Our investigation, however, was stimulated not by the points above but by concerns about the orbital angular momentum. As noted by Winn \& Holman (2005), the suggested tidal input of $\gtrsim 4 \times$ $10^{26} \mathrm{erg} \mathrm{s}^{-1}$ required to explain HD 209458b's present radius (Bodenheimer et al. 2003), if continued for $5 \mathrm{Gyr}$, is twice the present orbital energy of the planet. Hence, even if a somewhat smaller tidal power were acceptable or the planet suffered less dissipation in the past, the orbital energy must have changed by of order its present value, with a large loss of orbital angular momentum. Winn \& Holman (2005) have not explained where the angular momentum might have gone. It is not likely to have been absorbed by the star, for two reasons. First, the moment of inertia of the star is somewhat smaller than that of the orbit (\$3.3), so that, notwithstanding magnetic braking by a stellar wind, stars hosting inspiraling hot Jupiters might be expected to rotate more rapidly than other stars of similar age and spectral type, contrary to the evidence of Figure 3 in this paper. Second, the timescale for dissipating the planetary tide in the star probably exceeds its age (\$3.3), which limits the amount of angular momentum that the star can absorb. Without dissipation, the star can change only the direction but not the magnitude of its spin angular momentum 1 Thus, the amount of angular momentum that the star can absorb from the orbit is limited by the star's initial obliquity as well as its moment of inertia. In four cases where the spectroscopic transit (RossiterMcLaughlin effect) has been well measured, the implied angle $(\lambda)$ between the projections of the stellar spin and the orbital angular momentum onto the plane of the sky is small (Winn 2006, and references therein); in particular, $\lambda=-4.4 \pm 1.4$ for HD 209458 (Winn et al. 2005). Unless the stellar spin has a large component along the line of sight — which has been ruled out for HD 189733 at least (Winn et al. 2006b) - the smallness of $\lambda$ indicates that if maintenance of the Cassini state relies upon angular momentum changes in the star, then the future life expectancy of that state is much less than the present age of the system.

The plan of this paper is as follows. 92 presents a pedagogical review of Cassini states in general nondissipative, Hamiltonian systems. 3 discusses causes of the precession of the orbit in more specificity: either an oblate and oblique primary, or a third body. Tidal dissipation is introduced, and it is argued from empirical constraints on tidal quality factors that dissipation in the planet is more important than dissipation in the star for this problem. 44 presents and interprets the governing equations used to describe the tidal evolution of the planetary spin and orbit, which are adopted from Eggleton \& Kiseleva-Eggleton (2001) with small notational changes. In 95, we present exemplary numerical results and interpret them using analytic approximations. We explore how Cassini state 2 "breaks" due to the imbalance of dissipative and non-dissipative

\footnotetext{
${ }^{1}$ In the equilibrium tide, the tidal bulge raised in the star by the planet points directly at the planet, since the phase lag between the bulge and the perturber is proportional to the dissipation rate. Hence the planet exerts no torque on the tidal bulge, while any torque exerted on the star's rotational oblateness is perpendicular to the spin axis and therefore changes only the direction of the spin. Alternatively - and the following argument applies to dynamical as well as equilibrium tides - we may apply Kelvin's Circulation Theorem to a closed contour drawn along the rotational equator. The circulation $2 \pi R_{\star} v_{\text {rot }}$ is conserved in a nondissipative star, since $(\boldsymbol{\nabla} \times \boldsymbol{v}) \cdot \boldsymbol{\nabla} S$ is conserved following the fluid and we may take the curve to be the boundary of a surface of constant specific entropy $S$. A contradiction would therefore result if the equatorial velocity were to evolve secularly without dissipation.
} 
torques, and we find a strong constraint on the planetary dissipation rate that renders obliquity tides unimportant as an internal heat source. In $\$ 6$, we summarize our main results and use them to quantify the objections raised above to Cassini states as an explanation for the anomalously large radius of HD 209458b in particular.

\section{Cassini states}

The spin dynamics of a body in a precessing Keplerian orbit are analogous to orbital dynamics in a rotating 2D potential. In both systems a corotating frame is found in which the stationary points of the effective potential are equilibria. The spin counterparts to Lagrange points are Cassini states. In this section we review the theory of Cassini states.

The modern study of spin equilibria was begun by Colombo (1966), who generalized Cassini's laws for axisymmetric bodies. Peale (1969) first enumerated the Cassini states and generalized to the non-axisymmetric case. Initial applications of the theory were made primarily to Mercury and the moon, but also to Iapetus and Triton (Colombo 1966; Peale 1969, 1974; Beletskii 1972; Ward 1975). Evolution of the obliquity of Mars due to geophysical changes in the planet's oblateness was studied by Ward et al. (1979) and Henrard \& Murigande (1987). The latter work contains a particularly illuminating development of the Cassini formalism. Recent applications to Saturn (Ward \& Hamilton 2004) and Jupiter (Ward \& Canup 2006) suggest that the primordial obliquities of many solar system bodies may have been altered by trapping in Cassini resonances. Our present concern is with application to hot Jupiters (Winn \& Holman 2005; Levrard et al. 2007). The essential features of Cassini states may be derived by specializing to the case of a dissipationless, axisymmetric body. For a general treatment see Peale (1974). We discuss the critical role of tidal dissipation in $\$ 4.2$.

Consider an axisymmetric planet with principal moments of inertia $C>A=B$ in orbit around a star of mass $M_{\star}$. Let the total angular momentum of the system (including other bodies) be denoted $\mathbf{J}$, pointing along the fixed unit vector $\hat{\mathbf{J}}$. This axis and the invariable plane perpendicular to $\hat{\mathbf{J}}$ are at rest in an inertial frame. Let the orbital plane be inclined to the invariable plane by angle $I$, and allow the node to precess uniformly around $\hat{\mathbf{J}}$ with angular frequency $g$ due to an unspecified constant torque. In the two-body problem the nodal precession is dominated by the stellar quadrupole, but torques from additional bodies or the circumstellar disk may dominate when they are present. The orbit normal $\hat{\mathbf{h}}$ remains fixed in a frame rotating with angular velocity $\mathbf{g}=g \hat{\mathbf{J}}$ with respect to the inertial frame. In cases of interest the node regresses $(g<0)$. Define a Cartesian coordinate system $X Y Z$ attached to the rotating frame as in Figure 1, with the $Z$-axis along the orbit normal $(\hat{\mathbf{Z}}=\hat{\mathbf{h}})$ and the $X$-axis in the direction of the ascending node. The orientation of the planet relative to the rotating frame is specified by the usual Euler angles $(\theta, \phi, \psi)$, which give the positions of the planet's axis of symmetry $z$ and the arbitrary orthogonal axes $x$ and $y$ frozen into the body. The equatorial plane intersects the orbital plane at an obliquity $\theta=\cos ^{-1}(\hat{\mathbf{z}} \cdot \hat{\mathbf{h}})$. This node precesses around $\hat{\mathbf{h}}$ with angular frequency $\dot{\phi}$. The planet rotates with respect to the 
inertial frame with instantaneous angular velocity $\boldsymbol{\omega}$, which is the sum of the spin angular velocity $\boldsymbol{\Omega} \equiv \dot{\psi} \hat{\mathbf{z}}$ and the angular velocity associated with the precession of the symmetry axis. If the $z$-axis is at rest in the rotating frame then $\boldsymbol{\omega}=\boldsymbol{\Omega}+\mathbf{g}$. The planet's total spin angular momentum $\mathbf{S}=C \omega_{z} \hat{\mathbf{z}}+B \omega_{y} \hat{\mathbf{y}}+A \omega_{x} \hat{\mathbf{x}}$ can be approximated by principal axis rotation if the precessional angular frequency is small compared to $\Omega$, in which case only the $\hat{\mathbf{z}}$ component of $\mathbf{S}$ is retained. The further approximation $\omega_{z} \approx \Omega$ is often made for simplification, and we do so here. In Figure 1 we have implicitly assumed principal axis rotation by taking $\mathbf{S}=C \boldsymbol{\Omega}$. The Hamiltonian $\mathcal{H}_{\mathrm{RF}}$ governing the spin of the planet in the rotating frame is related to the Hamiltonian $\mathcal{H}_{\mathrm{IF}}$ in the inertial frame by $\mathcal{H}_{\mathrm{RF}}=\mathcal{H}_{\mathrm{IF}}-\mathbf{S} \cdot \mathrm{g}$. This is simply the relation between Jacobi's integral of motion in a rotating potential and the total energy. $\mathcal{H}_{\mathrm{IF}}$ is the total energy associated with the planet's orientation, and $\mathcal{H}_{\mathrm{RF}}$ is an integral of motion for constant $\mathbf{g}$ and in the absence of dissipation. Hereafter we drop the subscript RF. The Hamiltonian in the rotating frame

$$
\mathcal{H}=\frac{S^{2}}{2 C}+V-\mathbf{S} \cdot \mathbf{g}
$$

includes the orientation-dependent component $V$ of the planet's gravitational potential. This is contributed by spin-orbit coupling, while spin-spin coupling by the stellar quadrupole is negligible. Expanded in spherical harmonics and truncated at second order,

$$
V=\frac{G M_{\star}(C-A)}{r^{3}}\left[\frac{3}{2}(\hat{\mathbf{r}} \cdot \hat{\mathbf{S}})^{2}-\frac{1}{2}\right]
$$

where $\hat{\mathbf{r}}$ is the instantaneous direction of the planet with respect to the star and $r$ its distance. $V$ has a short-term variation over the orbit and a long-term variation with the spin precession. We may average $V$ over an orbital period while holding $\hat{\mathbf{S}}$ constant to obtain the mean potential on timescales much greater than the orbital period $P$. For an orbit with mean motion $n=2 \pi / P$ and eccentricity $e$,

$$
\frac{1}{P} \int_{0}^{P} V d t=-\frac{n^{2}(C-A)}{2\left(1-e^{2}\right)^{3 / 2}}\left[\frac{3}{2}(\hat{\mathbf{S}} \cdot \hat{\mathbf{h}})^{2}-\frac{1}{2}\right],
$$

which depends only on $\hat{\mathbf{S}}$ since $\hat{\mathbf{h}}$ is fixed in the rotating frame. The system described by (1) and (3) has 2 degrees of freedom. Its state may be specified by the generalized coordinates $(\psi, \phi)$ and conjugate momenta $(S, S \cos \theta)$. It is immediately apparent that $S$ is conserved due to the axisymmetry of the planet. We define the constants

$$
\alpha \equiv \frac{3 n^{2}(C-A)}{2 C \Omega\left(1-e^{2}\right)^{3 / 2}} \quad \text { and } \quad \mathcal{H}^{\prime} \equiv\left(\mathcal{H}-\frac{S^{2}}{2 C}\right) S^{-1}-\frac{\alpha}{6} .
$$

The former characterizes the natural precession frequency of the spin. The latter is the part of the Hamiltonian composed of variable terms:

$$
\mathcal{H}^{\prime}=-g(\hat{\mathbf{S}} \cdot \hat{\mathbf{J}})-\frac{\alpha}{2}(\hat{\mathbf{S}} \cdot \hat{\mathbf{h}})^{2} .
$$

Viewed in terms of the projection $\hat{\mathbf{S}}=(X, Y, Z)$ onto the rotating axes, equation (41) describes a family of parabolic cylinders opening in the $+Y$ direction (Colombo 1966). Solution trajectories 
for $\hat{\mathbf{S}}$ are curves of intersection between the parabolic cylinders and the unit sphere. The canonical equations of motion $\dot{\phi}=\partial \mathcal{H} / \partial(S \cos \theta)$ and $d(S \cos \theta) / d t=-\partial \mathcal{H} / \partial \phi$ become

$$
\frac{d \hat{\mathbf{S}}}{d t}=g(\hat{\mathbf{S}} \times \hat{\mathbf{J}})+\alpha(\hat{\mathbf{S}} \cdot \hat{\mathbf{h}})(\hat{\mathbf{S}} \times \hat{\mathbf{h}}) .
$$

The equilibrium solutions of (5) are Cassini states. Constant obliquity requires that the spin axis be coplanar with $\hat{\mathbf{J}}$ and the orbit normal (i.e. $\phi=0$ or $\pi$ ), because otherwise $\hat{\mathbf{S}} \times \hat{\mathbf{J}}$ and $\hat{\mathbf{S}} \times \hat{\mathbf{h}}$ point in different directions. This statement is Cassini's third law. Constant $\phi$ requires that the equilibrium obliquity $\theta$ satisfy a relation

$$
0=g \sin (\theta-I)+\alpha \cos \theta \sin \theta
$$

that has either two or four roots for given values of $g / \alpha$ and $I$. By convention $\theta$ is negative when $\phi=0$ and positive when $\phi=\pi$. Equation (6) is identical to that obtained by finding the stationary points of $\mathcal{H}^{\prime}$ on the unit sphere, which are points of tangency between the unit sphere and parabolic cylinders.

Figure 2 illustrates the equilibria admitted by equation (6) for $I=0.1$ (left panel) and $I=1.0$ (right panel). In the limit that the orbit precesses slowly $(|g / \alpha| \ll 1)$ the term derived from $V$ dominates the Hamiltonian. The mean gravitational potential is minimized when the spin is aligned or antialigned with the orbit normal (states 1 and 3, respectively), and maximized when the spin tips into the plane of the orbit, pointing in the $+Y$ direction (state 2) or the $-Y$ direction (state 4). States 1, 2, and 3 are stable against small displacements, which result in libration around the equilibrium point. The torque $\mathbf{S} \times \mathbf{g}$ is responsible for libration within a domain around state 2 , which would otherwise not be stable. State 4 is a saddle point of $\mathcal{H}^{\prime}$, lying on the trajectory that separates the domains of the other three states (i.e. the separatrix), and is therefore unstable. As $|g / \alpha|$ increases the domain of state 2 expands while that of state 1 contracts, until a critical point is reached at which states 1 and 4 merge and vanish. In the limit of a rapidly precessing orbit $(|g / \alpha| \gg 1)$ the planet responds to the time-average position of the orbit normal, namely $\hat{\mathbf{J}}$. $\mathcal{H}^{\prime}$ is then maximized for spin aligned with $\hat{\mathbf{J}}$ (state 2) and minimized for spin antialigned with $\hat{\mathbf{J}}$ (state 3). Comparison of the left and right panels illustrates that, at larger inclinations, the domain around state 2 is broader because it lies closer to $\hat{\mathbf{J}}$.

Winn \& Holman (2005) pointed out that Cassini state 2 is the most favorable configuration wherein a planet could maintain a significant obliquity. The obliquity of state 1 is large only for a coincidental similarity of $g$ and $\alpha$ (near the critical point), which are unrelated parameters. As

for state 3 , we show in 4.1 that no such equilibrium exists in the presence of a dissipative tidal torque.

\section{Tidal interactions}

As we have just seen, a torque must be exerted on the planetary orbit to keep it in Cassini state 2. This torque could be tidal, involving the oblateness and obliquity of the central star, or 
it could be due to a third body in an inclined orbit. We examine both possibilities below. Tidal dissipation must occur in the planet at some level if it remains in Cassini state 2. We compare the relative importance of dissipation in the planet and in the star for the evolution of the orbital energy and angular momentum.

\subsection{Orbit precessed by stellar oblateness}

Given dissipation in a body, the spin will move towards alignment with the orbit normal. However, given reasonable values for the star's energy dissipation rate, the tide of a hot Jupiter is too weak to cause the angle between the star's spin and its orbit to change substantially (the time scale for such change is similar to equation (13) below). Therefore, measurements of alignment among extrasolar planets (Queloz et al. 2000; Winn et al. 2005; Winn et al. 2006a) are understood to be faithful indicators of the primordial inclination. A system that is not perfectly aligned will undergo precession analogous to that of the Earth's spin as the Earth's rotational bulge is torqued by both the moon and the sun. As an extrasolar planet causes its host star to precess, the planet's orbit also precesses, conserving total angular momentum. By this mechanism, the orbits of hot Jupiters may precess at rates that are fast enough to be observable by transit timing (Miralda-Escudé 2002). In such systems, the star's spin angular momentum $\mathbf{S}_{\star}=C_{\star} \boldsymbol{\Omega}_{\star}$ has a magnitude comparable to, but somewhat smaller than, that of the orbital angular momentum $\mathbf{L}$, and the planet's spin angular momentum $\mathbf{S}_{\mathbf{p}}=C_{p} \boldsymbol{\Omega}_{\mathbf{p}}$ has negligible magnitude. The total angular momentum $\mathbf{J}=\mathbf{L}+\mathbf{S}_{\star}+\mathbf{S}_{\mathbf{p}}$ is located between the stellar spin and orbit normal, but it lies slightly outside of the plane defined by $\mathbf{L}$ and $\mathbf{S}_{\star}$ unless $\mathbf{S}_{\mathbf{p}}$ lies in that plane. If the time average of $\mathbf{S}_{\mathbf{p}}$ is kept out of that plane, then the misalignment between stellar spin and the orbit normal can evolve, even without dissipation in the star (see \$5).

\subsection{Orbit precessed by third body}

Another source of orbital precession is a third body. There are two known transiting planets in binaries (HD 189733b \& HAT-P-1b), but the stellar companions would cause planetary orbital precession on a timescale much longer than the host star's stellar oblateness would, so their effect is negligible. No system with a transiting planet has a known second planet.

Suppose there is an undetected second planet on an exterior orbit. It will perturb the eccentricity of the transiting planet on a short timescale, which will be continually damped by solar tides, which would also heat the planet. However, as the transiting planet's orbit shrinks due to this continual excitation and damping of eccentricity, the rate $|g|$ at which it is precessed by the exterior planet will decrease. Also, as the orbit shrinks, $\alpha$ increases. If the planet started with

$|g| / \alpha>(|g| / \alpha)_{\text {crit }}$, Cassini state 2 was the only equilibrium available. As $|g| / \alpha$ slowly drops, the spin axis will tip over as $|g| / \alpha$ crosses $(|g| / \alpha)_{\text {crit }}$ and the spin remains in state 2 (see Fig. (2). There- 
fore a mixture of eccentricity and obliquity heating is possible, if Cassini states are stable despite dissipation.

The amount of angular momentum that the second planet can absorb from the first as its orbit shrinks can constrain the parameters of the second planet. Let $L$ and $L_{2}$ be the orbital angular momentum of the transiting planet and the second planet, respectively. Suppose that $L$ and $L_{2}$ are the dominant angular momenta of the problem such that, by the law of cosines,

$$
J^{2}=L^{2}+L_{2}^{2}+2 L L_{2} \cos i_{\text {rel }},
$$

where $i_{\text {rel }}$ is the relative inclination of the two planets' orbits. The semimajor axis and eccentricity of the second planet are both conserved on secular timescales if the semimajor axis ratio is large, which can be shown by taking the potential of the second planet to quadrupole order and integrating over the orbits of both planets (Ford et al. 2000). Therefore, the only way the orbit of a second planet can absorb angular momentum from the dissipating planet is by reorienting. As the planets approach coplanarity, the orbital precession period becomes too long for the dissipating planet to remain in Cassini state 2. The obliquity damps to zero and loss of energy and angular momentum halts. For the final condition of $i_{\text {rel }}=0$, we therefore have $J=L_{f}+L_{2}$, where $L_{f}$ is the final angular momentum of the dissipating planet. Constancy of $J$ gives:

$$
L_{f}=\left(L_{i}^{2}+L_{2}^{2}+2 L_{i} L_{2} \cos i_{\mathrm{rel}, \mathrm{i}}\right)^{1 / 2}-L_{2},
$$

where $L_{i}$ and $i_{\text {rel, }}$ are the initial angular momentum of the dissipating planet and the initial relative inclination, respectively. Solving for the angular momentum of the second planet gives

$$
L_{2}=\frac{1}{2} \frac{L_{i}^{2}-L_{f}^{2}}{L_{f}-L_{i} \cos i_{\mathrm{rel}, \mathrm{i}}} .
$$

For any solution to exist, we must have $i_{\text {rel,i }}>i_{\text {rel,crit }}=\cos ^{-1}\left(L_{f} / L_{i}\right)$. This critical value corresponds to the case in which $L_{2} \gg L$ and essentially all of the reorienting is done by $L$.

If we suppose that a particular planet is currently in Cassini state 2, with its semimajor axis still shrinking, then $L_{\text {now }}>L_{f}$. If a second planet is the cause of orbital precession, its angular momentum must be greater than $L_{2}$ of equation (9), taking $L_{f} \rightarrow L_{\text {now }}$. This constraint may be very restrictive, for a presumed value of $i_{\text {rel,i, }}$, if we can estimate $L_{i}$. Tidal dissipation is a strong function of semi-major axis; from the equations of $\$ 4$ one may derive an approximate relation $\dot{L} \propto-L^{-12}$. If the rate of angular momentum evolution $\dot{L}$ is observed or is theoretically inferred (for instance, by an inflated radius), this relation can be integrated back in time for the age of the

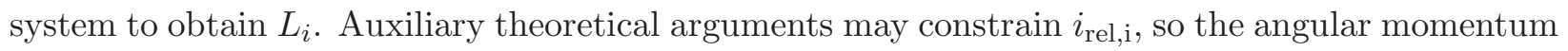
$L_{2}$ of an undetected second planet may be given a lower bound, if it is forcing obliquity tides. The example of HD 209458b will be examined in $₫ 6$. 


\subsection{Dissipation}

The nature of tidal dissipation in Jovian planets and late-type main-sequence stars remains a subject of research. Such bodies are largely fluid, and the tidal Reynolds number based on the true microscopic viscosity is enormous. Therefore, most theories for the dissipation invoke resonant coupling to stellar convection (Zahn 1966), or to modes of oscillation (Zahn 1970; Ogilvie \& Lin 2004; Wu 2005). Where these mechanisms can be worked out with confidence, they often fall short of explaining observationally inferred rates of circularization or synchronization. Other mechanisms show promise but require difficult and uncertain calculations. Therefore, in the present work, we rely upon empirical scalings from observations. Following Goldreich \& Soter (1966, henceforth GS66), we parametrize the dissipation by a tidal quality factor $Q$ defined as the ratio of the maximum stored energy in the tidal distortion of the star to the energy dissipated per radian of the tidal cycle. The "equilibrium tide" approximation is used, as if the tide were static, so the stored energy is potential. In this formulation, the phase lag between the applied tidal potential and the response is $(2 Q)^{-1}$. The torque on a nonrotating body of mass $M_{1}$ and radius $R_{1}$ due to a companion of mass $M_{2}$ at separation $r$ is

$$
\Gamma_{1}=\frac{3 k_{1}}{Q_{1}} \frac{G M_{2}^{2} R_{1}^{5}}{r^{6}} .
$$

The quantity $k_{1}$ is the apsidal-motion constant (which is half the Love number), which GS66 effectively replace by $3 / 4$ as appropriate for a body of uniform density - a reasonable approximation for a solid planet or moon. The structures of solar-type stars and Jovian planets are better approximated by polytropes of indices $n_{\star}=3$ and $n_{p}=1$, respectively, for which $\left(k_{\star}, k_{p}\right) \approx(0.014,0.26)$. In all tidal dissipation rates, however, $Q$ and $k$ appear in the same combination that one sees in equation (10) above, so it is convenient to frame the discussion in terms of an effective quality factor $2 Q^{\prime} \equiv Q \times 3 /(4 k)$.

Values for the quality factor can be inferred from orbital properties of appropriate binary stars, extrasolar planets, and satellites of Solar System planets. Since it is simply a parametrization of ill-understood dissipative processes, $Q^{\prime}$ may depend upon tidal period, and also on subtle details of internal structure. As an example of the latter, Ogilvie \& Lin (2004) state that their inertialwave mechanism depends upon the existence of a rocky planetary core; there is evidence from the measured radii of transiting planets that their cores vary considerably in size and mass.

With these caveats in mind, we review some of the observational inferences regarding $Q^{\prime}$. GS66 estimated that the mean-motion resonances of Io, Europa, and Ganymede require Jupiter's quality factor to lie in the range $10^{5} \lesssim Q_{J}^{\prime} \lesssim 10^{6}$, unless that resonance is accidental or short-lived. The relevant tidal period in this case is $6.5 \mathrm{hr}$, one half the synodic period between Jupiter's rotation and Io's revolution. For spectroscopic binary stars, tidal circularization is easier to recognize than tidal synchronization, since orbital eccentricity is measurable from the radial-velocity curve.

\footnotetext{
${ }^{2}$ In GS66's original notation, $Q$ and $Q^{\prime}$ differ by a term involving elasticity, which can be significant for solid planets but is presumed to be negligible in our case. Our $Q^{\prime}$ reduces to their $Q$ for a uniform-density body.
} 
Mathieu et al. (1992) and Mathieu et al. (2004, and references therein) find that the circularization period $\left(P_{\text {circ }}\right)$ separating circular from measurably eccentric binary orbits in stellar clusters increases with cluster age beyond $1 \mathrm{Gyr}$, indicating that significant tidal dissipation occurs on the main sequence. For M67 and NGC 188, for example, $P_{\text {circ }}=12.4 \mathrm{~d}$ and $P_{\text {circ }}=15 \mathrm{~d}$ are quoted, with corresponding cluster ages of 5 and 7 Gyr, respectively, whereas clusters younger than 1 Gyr are said to be consistent with $P_{\text {circ }} \approx 8$ d, perhaps reflecting rapid circularization during protostellar phases (Zahn \& Bouchet 1989). To relate such observations to tidal dissipation rates, it is conventional to suppose that $P_{\text {circ }}$ is the period at which eccentricity has decayed by three e-foldings. Adapting GS66's formula for the damping rate due to star 1 alone, we have

$$
\left(\frac{d \ln e}{d t}\right)_{1}=-\frac{171}{16 Q^{\prime}} \frac{M_{2}}{M_{1}}\left(\frac{R_{1}}{a}\right)^{5} n
$$

in which $a$ is the binary semimajor axis, $n=\left(G M_{\text {tot }} / a^{3}\right)^{1 / 2}$ is the mean motion of the orbit, and synchronous rotation has been assumed. Applying this to a binary consisting of two sunlike stars and summing the dissipation rates in both stars, we estimate from M67 that $Q_{\odot}^{\prime} \approx 3.5 \times 10^{5}$, and from NGC 188 that $Q_{\odot}^{\prime} \approx 2.2 \times 10^{5}$.

The fact that the orbits of most extrasolar planets with periods less than $\sim 5$ days and masses $\gtrsim M_{J}$ are essentially circular provides another constraint on $Q_{p}^{\prime}$, if we assume that this is due to tidal dissipation in the planet 3 Again taking $|d \ln e / d t|^{-1}=t / 3$ at $P_{\text {circ }}$ and using $t=5$ Gyr as a representative age, one has

$$
Q_{p}^{\prime} \approx 3 \times 10^{5}\left(\frac{P_{\text {circ }}}{5 \mathrm{~d}}\right)^{-13 / 3}\left(\frac{M_{\star}}{M_{\odot}}\right)^{-2 / 3}\left(\frac{M_{J}}{M_{p}}\right)\left(\frac{R_{p}}{R_{J}}\right)^{5} .
$$

Alternatively, circularization might be due to dissipation in the star. However, equation (11) predicts that $d \ln e / d t$ is dominated by whichever body has the smaller value of $Q^{\prime} M^{2} / R^{5}$. This is probably the planet, as discussed below.

It is reassuring that these various estimates for $Q^{\prime}$ in Jupiter, extrasolar planets, and sunlike stars are comparable; in fact, one might boldly conclude from the evidence above that there is a universal value $Q^{\prime} \sim 3 \times 10^{5}$. Many theories of tidal dissipation, however, predict that $Q^{\prime}$ should scale with tidal period. For equilibrium tides subject to a frequency-independent effective viscosity (perhaps associated with turbulent convection), it can be shown that $Q^{\prime} \propto P$. With this scaling, the values estimated for Jupiter from its Galilean moons should be scaled up by an order of magnitude for extrasolar planets in $\sim 4$ d orbits. Goldreich \& Nicholson (1977) argued that when the tidal period is less than the turnover time of the energy-bearing convective eddies, the effective viscosity they provide should be suppressed by a factor $\sim\left(P_{\text {tide }} / T_{\text {eddy }}\right)^{2}$; this would

\footnotetext{
${ }^{3}$ At the time of writing, based on the catalog at http://vo.obspm.fr/exoplanetes/encyclo, we find $P_{\text {circ }} \gtrsim 5 \mathrm{~d}$. GJ $436 \mathrm{~b}$ is the only planet with $P<5 \mathrm{~d}$ that has an eccentricity securely above 0.1 , which Maness et al. (2007) attribute to either ongoing eccentricity pumping or an unusually high $Q$ for this rather low $\operatorname{mass}\left(M \sin i \approx 23 M_{\oplus}\right)$ planet.
} 
lead to a quality factor decreasing with increasing period as $Q^{\prime} \propto P^{-1}$. Goldman \& Mazeh (1991) suggested that this scaling fits the observed trend of binary circularization periods with age better than an increasing or constant $Q^{\prime}$. However, if the scaling $Q^{\prime} \propto P^{-1}$ results from a suppression of convective dissipation at short periods, then it is very unlikely that convection can account for the inferred $Q^{\prime}$ of Jupiter at all, as Goldreich \& Nicholson (1977) point out. The inertial-wave mechanism of Ogilvie \& Lin (2004), on the other hand, apparently predicts a quality factor that is independent of tidal frequency for a fixed ratio between the tidal and rotation frequencies.

In the case of hot Jupiters, dissipation in the planet is likely to be more important than dissipation in the star. On the one hand, it follows from equation (10) that the ratio of the synchronization torques on the spins of the two bodies is

$$
\frac{\Gamma_{\star}}{\Gamma_{p}}=\frac{Q_{p}^{\prime}}{Q_{\star}^{\prime}} \times \frac{\bar{\rho}_{p}^{2} R_{p}}{\bar{\rho}_{\star}^{2} R_{\star}},
$$

where $\bar{\rho}_{i} \equiv 3 M_{i} / 4 \pi R_{i}^{3}$ is the average density of body $i$. The same ratio determines the relative importance of the two bodies for eccentricity damping. The densities of the star and planet are typically comparable, and the mass ratio $M_{p}: M_{\star} \sim 10^{-3}$, so the second factor on the right hand side above is $\lesssim\left(M_{p} / M_{\star}\right)^{1 / 3} \sim 0.1$. So the torque on the planet is greater unless the star has a substantially smaller $Q^{\prime}$. On the other hand, the synchronization timescale due to the torque on the star is approximately

$$
t_{\mathrm{sync}, \star} \equiv \frac{\left(M_{p} a^{2}+C_{\star}\right)}{\Gamma_{\star}}\left|n \hat{\mathbf{h}}-\Omega_{\star}\right| \approx 15 \frac{Q_{\star}^{\prime}}{3 \times 10^{5}}\left(\frac{P}{4 \mathrm{~d}}\right)^{13 / 3}\left(\frac{\bar{\rho}_{\star}}{\bar{\rho}_{\odot}}\right)^{5 / 3}\left(\frac{M_{J}}{M_{p}}\right) \mathrm{Gyr},
$$

in which $C_{\star} \approx 0.06 M_{\star} R_{\star}^{2}$ is the stellar moment of inertia. Note that the stellar and orbital angular velocities are differenced as vectors, to allow for possible stellar obliquity. In the final numerical form, we have neglected $\Omega_{\star}$ compared to $n \hat{\mathbf{h}}$ and taken $C_{\star} \approx M_{p} a^{2}$ : in fact $C_{\star} /\left(M_{p} a^{2}\right) \approx$ $0.6(P / 4 \mathrm{~d})^{-4 / 3}$ for a sunlike star and a Jupiter-mass planet. Thus, the stellar spin will not have been substantially altered by its tidal interaction with the planet unless $Q_{\star}^{\prime}<10^{5}$, contrary to the evidence of binary stars. Of course, $Q_{\star}^{\prime}$ may depend upon the companion. Theory suggests that $Q_{\star}$ may be greater under the tidal influence of a planetary rather than a stellar companion (Ogilvie \& Lin 2007).

Moreover, the spectroscopic $(v \sin i)_{\star}$ values of exoplanetary hosts indicate that the stars rotate subsynchronously. To emphasize this point, in Figure 3 we plot the values of $(v \sin i)_{\star}$, relative to the synchronous value, for the known transiting planets. The value of $\sin i$ should be very close to 1 if there is approximate alignment between stellar spin and planet orbit, as has been observed in four cases (Winn 2006, and references therein). All of the stars rotate much slower than their planet's orbit. In fact, their spin rates are largely consistent with a standard Skumanich law - an empirical relation $v \propto t^{-0.5}$ that describes the braking with age $t$ of late-type dwarf stars due to the stellar wind (Skumanich 1972). A suitable reference for calibrating the Skumanich law is Valenti \& Fischer (2005)'s spectroscopic catalogue of 1040 nearby F, G, and K stars that have been targeted by planet 
searches. We calibrate separately for six mass bins, the first with $0.7 \leq M_{\star} / M_{\odot}<0.9$, and the remaining five bins spaced between $0.9-1.4 \mathrm{M}_{\odot}$, each with width $0.1 \mathrm{M}_{\odot}$. In all bins a better fit to the data can be obtained with a power law steeper than -0.5 , but we ignore this systematic and depict standard Skumanich laws for simplicity. Scatter around the fits is $\approx 10 \%$. Only HD 209458 appears to be rotating more rapidly than would be predicted by magnetic braking alone (and only marginally so, given the scatter and systematic uncertainties). The tidal torque these stars receive from their planets is apparently weak compared to the torque from their magnetospheres. Stars HAT-P-1 and TrES-1 are the relatively slow rotators of the group; however, note that on this figure, the Sun would also be rotating slowly by a factor of 2 .

We conclude that for the purposes of the present paper, tidal dissipation in the host star can be neglected.

\section{Spin and orbit equations of motion}

Here we introduce the equations of Eggleton \& Kiseleva-Eggleton (2001) for the spin and orbit evolution of two fluid bodies. The equations of motion are for the orbital elements in the secular approximation. That is, the Newtonian equations of motion are integrated over one orbit, and what remains is time derivatives for the orbital elements. This formulation allows us to perform integrations quickly that represent dynamical evolution over billions of years. The parameters of energy dissipation in this model are based on the assumption that the energy loss rate is proportional to the time rate of change of the quadrupole tensor (Eggleton et al. 1998). A prolate bulge is raised on each body by the tidal potential of its companion. If the rotation is not synchronized, energy dissipation drags the bulge away from the instantaneous direction of the companion. The familiar consequences are that the spins of the bodies synchronize with the orbit and that the orbit's eccentricity is damped as its energy is sapped at nearly constant angular momentum. Another effect quantified by this model is the spins coming into alignment with the orbit normal, which is the key piece of technology we need to investigate the relationship of tidal synchronization to Cassini states.

We shall specialize to the limit of zero orbital eccentricity, for a few reasons. First, the eccentricity will damp to zero on a timescale short compared to the age of the system. Second, the effect of eccentricity damping in hot Jupiters has been pursued by other authors (Bodenheimer et al. 2001; Levrard et al. 2007), so this approximation more clearly focuses attention on the contribution of obliquity to tidal dissipation. Finally, this approximation considerably simplifies the equations of Eggleton \& Kiseleva-Eggleton (2001); for instance, the only included effect of general relativity is to cause the apse to precess, which has no physical effect when $e=0$.

Let the two masses equal $M_{\star}$ and $M_{p}$, of total mass $M$, and of reduced mass $\mu=M_{\star} M_{p} / M$. Let $\mathbf{h}$ denote the orbital angular momentum per unit reduced mass, of magnitude $h$ and direction $\hat{\mathbf{h}}$, and let $\hat{\mathbf{e}}$ and $\hat{\mathbf{q}}$ be reference directions in the plane of the orbit such that $\hat{\mathbf{e}} \times \hat{\mathbf{q}}=\hat{\mathbf{h}}$. Let 
the vector spins of the two bodies be $\boldsymbol{\Omega}_{\star}$ and $\boldsymbol{\Omega}_{\mathbf{p}}$ with rotational moments of inertia $C_{\star}$ and $C_{p}$. Therefore, with our specialization to $e=0$, there are 9 dynamical variables: the 9 components of the vectors $\mathbf{h}, \boldsymbol{\Omega}_{\star}$, and $\boldsymbol{\Omega}_{\mathbf{p}}$. The equations conserve the total angular momentum. The equations of motion are:

$$
\begin{aligned}
\frac{1}{h} \frac{d \mathbf{h}}{d t} & =\left(\mathcal{Y}_{\star}+\mathcal{Y}_{p}\right) \hat{\mathbf{e}}-\left(\mathcal{X}_{\star}+\mathcal{X}_{p}\right) \hat{\mathbf{q}}-\left(\mathcal{W}_{\star}+\mathcal{W}_{p}\right) \hat{\mathbf{h}} \\
C_{i} \frac{d \boldsymbol{\Omega}_{\mathbf{i}}}{d t} & =\mu h\left(-\mathcal{Y}_{i} \hat{\mathbf{e}}+\mathcal{X}_{i} \hat{\mathbf{q}}+\mathcal{W}_{i} \hat{\mathbf{h}}\right),
\end{aligned}
$$

where

$$
\begin{aligned}
\mathcal{X}_{i} & =-k_{i} \frac{M}{M_{i}} \frac{1}{n}\left(\frac{R_{i}}{a}\right)^{5} \Omega_{i h} \Omega_{i e}-\frac{\Omega_{i q}}{2 n t_{F i}} \\
\mathcal{Y}_{i} & =-k_{i} \frac{M}{M_{i}} \frac{1}{n}\left(\frac{R_{i}}{a}\right)^{5} \Omega_{i h} \Omega_{i q}+\frac{\Omega_{i e}}{2 n t_{F i}} \\
\mathcal{W}_{i} & =\frac{1}{t_{F i}}\left(1-\frac{\Omega_{i h}}{n}\right),
\end{aligned}
$$

and $i$ is either $p$ or $\star$. $\quad R_{i}$ is the radius of each body, $a=h^{2} /(G M)$ is the semi-major axis, $n=(G M)^{2} h^{-3}$ is the mean motion, $k_{i}$ is the apsidal motion constant, and $t_{F i}$ is a tidal friction timescale:

$$
t_{F i}=\frac{4 Q_{i}^{\prime}}{9}\left(\frac{a}{R_{i}}\right)^{5} \frac{M_{i}^{2}}{\mu M} n^{-1} .
$$

The integrations in $\$ 5$ hold the product $Q_{i}^{\prime} n^{-1}$ constant during the evolution, as recommended by the equilibrium tide model of Eggleton et al. (1998).

\subsection{Equilibration}

In equations (16) and (17), the first term on the right hand side is a non-dissipative torque that gives rise to nodal precession of the spins and orbit. The second term in these equations and the term on the right hand side of equation (18) are due to tidal dissipation. Since dissipation in the star is negligible, we may set $t_{F \star} \rightarrow \infty$, and specialize the discussion to dissipative torques on the planet.

In equilibrium the magnitude $\Omega_{p}$ of the planetary spin angular velocity is constant. Therefore

$$
\left\langle\boldsymbol{\Gamma} \cdot \boldsymbol{\Omega}_{p}\right\rangle=0,
$$

where $\boldsymbol{\Gamma}$ is the total torque on the planetary spin, and the angular brackets denote an average over the orbital mean motion. It suffices to replace $\boldsymbol{\Gamma}$ by the tidal (i.e. dissipative) component, since the precessional (i.e. nondissipative) part already satisfies (20). In the equilibrium tide, the instantaneous tidal torque takes the form

$$
\Gamma_{t}=-\kappa \hat{\boldsymbol{r}} \times(\hat{\boldsymbol{r}} \times \boldsymbol{u}),
$$


where $\boldsymbol{u} \equiv n \hat{\boldsymbol{h}}-\boldsymbol{\Omega}_{p}$ and $\hat{\boldsymbol{r}}$ is a unit vector from the star toward the planet. The logic behind equation (21) is that if the tidal bulge is a prolate distortion with major axis direction $\hat{\boldsymbol{b}}$, then $\boldsymbol{\Gamma}_{t} \propto \hat{\boldsymbol{b}} \times \hat{\boldsymbol{r}}$. Absent dissipation, $\hat{\boldsymbol{b}}=\hat{\boldsymbol{r}}$ so that the torque vanishes. Dissipation gives the bulge a phase lag of magnitude $\approx(2 Q)^{-1}$ with a direction determined by the angular velocity of the tide seen in a frame spinning with the planet. This angular velocity is $\boldsymbol{u}$, so that $\hat{\boldsymbol{b}} \approx \hat{\boldsymbol{r}}+(2 Q)^{-1}(\hat{\mathbf{r}} \times \mathbf{u}) /|\hat{\mathbf{r}} \times \mathbf{u}|$. Comparison with the equations of motion shows that $\kappa=\mu h\left(n t_{F p}\right)^{-1}$. Substituting equation (21) into equation (20) gives

$$
\boldsymbol{\Omega}_{p} \cdot(\boldsymbol{u}-\boldsymbol{u} \cdot\langle\hat{\boldsymbol{r}} \hat{\boldsymbol{r}}\rangle)=0,
$$

since the precession of $\Omega_{p} \& \boldsymbol{u}$ can be neglected on orbital timescales. Note that $\kappa$, which measures the strength of the tide, drops out. Now it is easy to show that $\langle\hat{\boldsymbol{r}} \hat{\boldsymbol{r}}\rangle=\frac{1}{2}(\mathbf{I}-\hat{\boldsymbol{h}} \hat{\boldsymbol{h}})$, which, in combination with (22), yields $\boldsymbol{\Omega}_{p} \cdot \boldsymbol{u}+\left(\boldsymbol{\Omega}_{p} \cdot \hat{\boldsymbol{h}}\right)(\boldsymbol{u} \cdot \hat{\boldsymbol{h}})=0$. Solving for the equilibrium spin frequency of the planet, we have the correct statement of Cassini's first law:

$$
\Omega_{p}=\frac{2 n}{\cos \theta+\sec \theta}
$$

This is the exact solution of the equations of motion when $\dot{\Omega}_{p}=0$. No equilibrium exists for $\theta>\pi / 2$; in particular, Cassini state 3 becomes unstable in the presence of the dissipative torque. Generally the synchronization time is comparable to the parallelization time (as we show next), so $\Omega_{p} \rightarrow n$ as $\theta \rightarrow 0$, but if there is a mechanism for maintaining non-zero obliquity, like trapping in Cassini state 2, $\Omega_{p}<n$. Equation (23) can be understood as pseudo-synchronization when obliquity is held at a constant value; an oblique and tidally evolved planet can be expected to be a slow rotator. These points were made by Levrard et al. (2007), who gave an expression for $\Omega_{p}$ with non-zero eccentricity (the dependence on $\theta$ is separable from the dependence on $e$ ).

Neglecting orbital precession, the dissipative torque from equations (15)-(17) will cause parallelization; the component of the spin perpendicular to the orbit $\left(\Omega_{p \perp}\right)$ decays approximately exponentially on a timescale $\tau_{\perp}=2 C_{p} n(\mu h)^{-1} t_{F p}$. The deviation from synchronization $\left(\Omega_{p h}-n\right)$ also decays, but on a timescale $\tau_{s}=\frac{1}{2} \tau_{\perp}$. The twice longer timescale for parallelization is because the parallelization torque takes on smaller values in parts of the orbit, whereas the synchronization torque is constant. For precessing orbits that trap the spin in Cassini state 2, equation (23) applies after a few parallelization times $\tau_{\perp}$, although it has no dependence on the tidal dissipation rate.

\subsection{Cassini states with dissipation}

The Cassini state formalism may be generalized to include ongoing tidal torques. For the generalized Cassini state we require that all the angular momenta vectors are stationary with respect to each other, even as the whole system precesses uniformly about the $\mathbf{J}$ axis. Cassini

\footnotetext{
${ }^{4}$ By symmetry, $\langle\hat{\boldsymbol{r}} \hat{\boldsymbol{r}}\rangle$ annihilates $\hat{\boldsymbol{h}}$ and is a multiple of the identity I for any vector lying in the orbital plane. The factor $(1 / 2)$ follows by considering the orbit average of $(\boldsymbol{d} \cdot \hat{\boldsymbol{r}})^{2}$ for any constant vector $\boldsymbol{d}$ in the plane.
} 
states are defined on timescales over which the orbital precession frequency $g$ is nearly constant; on tidal dissipation timescales, $g$ varies and the state evolves.

In the presence of dissipation, $\Omega_{\mathbf{p}}$ is shifted from the usual Cassini state; it does not lie in the plane defined by $\mathbf{h}$ and $\boldsymbol{\Omega}_{\star}$. The reason is that dissipation introduces terms that attempt to align the planet's spin with its orbit, which would cause $\boldsymbol{\Omega}_{\mathbf{p}}$ to slowly spiral in towards $\mathbf{h}$. In particular, the second terms of equations (16) and (17) cause a damping of the $e$ - and $q$-components of the spin, according to equation (15). The system is able to avoid this drift by readjusting to a state in which the planetary spin executes pure precession about $\mathbf{J}$. If the planet's spin and the orbit normal are stationary in a frame precessing with uniform angular velocity $\mathbf{g}=g \hat{\mathbf{J}}$, then $\dot{\Omega}_{\mathbf{p}}=\mathbf{g} \times \boldsymbol{\Omega}_{\mathbf{p}}$ and $\dot{\mathbf{h}}=\mathbf{g} \times \mathbf{h}$. Three constraints are needed on the three components of $\boldsymbol{\Omega}_{\mathbf{p}}$.

To analyze the situation, let us align the reference direction $\hat{\mathbf{q}}$ such that $\mathbf{J}$ lies in the $\hat{\mathbf{q}}-\hat{\mathbf{h}}$ plane, making an angle $I$ with the orbit normal $\hat{\mathbf{h}}$ as in Figure 1 (i.e. $\hat{\mathbf{q}}=\hat{\mathbf{Y}}$ and $\hat{\mathbf{e}}=\hat{\mathbf{X}})$. Anticipating that Cassini state 2 will be shifted out of the $\hat{\mathbf{q}}-\hat{\mathbf{h}}$ plane, denote the phase shift angle $\phi_{s} \equiv \pi-\phi$, as it is the supplement of $\phi$. Let the angle between $\boldsymbol{\Omega}_{\mathbf{p}}$ and $\mathbf{h}$ be denoted $\theta$ and the angle between $\boldsymbol{\Omega}_{\star}$ and $\mathbf{h}$ be denoted $\theta_{\star}$. Then, in the eqh coordinate system, we have

$$
\begin{aligned}
\mathbf{g} & =g(0, \sin I, \cos I), \\
\boldsymbol{\Omega}_{\mathbf{p}} & =\Omega_{p}\left(\sin \phi_{s} \sin \theta, \cos \phi_{s} \sin \theta, \cos \theta\right), \\
\dot{\Omega}_{\mathbf{p}} & =g \Omega_{p}\left(\cos \phi_{s} \sin \theta \cos I-\cos \theta \sin I,-\sin \phi_{s} \sin \theta \cos I, \sin \phi_{s} \sin \theta \sin I\right) .
\end{aligned}
$$

Note that $g<0$ because the nodes regress. Equations (15), (16), and (17) give the ratio between $\left(\dot{\boldsymbol{\Omega}}_{\mathbf{p}}\right)_{e}$ and $\left(\dot{\boldsymbol{\Omega}}_{\mathbf{p}}\right)_{q}$. Equating that to the ratio given by (26), we obtain the first constraint:

$$
\begin{aligned}
\frac{-\cos \phi_{s} \cos \theta+\xi^{-1} \sin \phi_{s}}{\sin \phi_{s} \cos \theta+\xi^{-1} \cos \phi_{s}} & =\frac{-\cos \phi_{s} \sin \theta \cos I+\cos \theta \sin I}{\sin \phi_{s} \sin \theta \cos I}, \\
\text { or equivalently, } \quad \sin \left(\delta+\phi_{s}\right) / \sin (\delta) & =\tan \theta \cot I,
\end{aligned}
$$

where

$$
\xi \equiv 2 k_{p} \Omega_{p} t_{F p} \frac{M}{M_{p}}\left(\frac{R_{p}}{a}\right)^{5}=\frac{2}{3} Q_{p} \frac{\Omega_{p}}{n} \frac{M_{p}}{\mu} \quad \text { and } \quad \tan \delta \equiv(\xi \cos \theta)^{-1} .
$$

Note that $\xi$ has been written in terms of $Q_{p}$ rather than $Q_{p}^{\prime}=3 Q_{p} /\left(4 k_{p}\right)$; nevertheless, it is likely to be quite large.

The second constraint, similarly obtained from the ratio between $\left(\dot{\Omega}_{\mathbf{p}}\right)_{h}$ and $\left(\dot{\Omega}_{\mathbf{p}}\right)_{q}$, gives an expression for the equilibrium spin rate that we have already derived in equation (23). The final constraint can be derived by setting equal the precession rates of the planet's orbit and spin. The former (from Eq. 14) is essentially due to the stellar oblateness since the rotational angular momentum of the planet is negligible $\left(\mathcal{X}_{p} \ll \mathcal{X}_{\star}\right.$, etc.):

$$
\begin{aligned}
g & =-\frac{1}{\sin I} \frac{|\dot{\mathbf{h}}|}{|\mathbf{h}|} \\
& =-k_{\star} \frac{M}{M_{\star}}\left(\frac{R_{\star}}{a}\right)^{5} \frac{\Omega_{\star}^{2}}{n} \frac{\sin \theta_{\star} \cos \theta_{\star}}{\sin I} .
\end{aligned}
$$


The planet's spin precesses about the total angular momentum, maintaining with it an angle $\beta \equiv \cos ^{-1}\left(\hat{\mathbf{J}} \cdot \hat{\Omega}_{\mathbf{p}}\right)$, at the rate

$$
\begin{aligned}
g & =-\frac{1}{\sin \beta} \frac{\left|\dot{\boldsymbol{\Omega}}_{\mathbf{p}}\right|}{\left|\boldsymbol{\Omega}_{\mathbf{p}}\right|} \\
& =-\frac{\mu h}{C_{p}} \frac{\sin \theta}{\sin \beta}\left\{\left[\frac{M}{M_{p}}\left(\frac{R_{p}}{a}\right)^{5} \frac{2 k_{p}}{1+\sec ^{2} \theta}\right]^{2}+\left[\frac{1}{2 n t_{F p} \cos \theta}\right]^{2}\right\}^{1 / 2} .
\end{aligned}
$$

Solutions of equations (23), (27), (28), and (29) are generalized Cassini states.

Given the complexity, solutions for $\phi_{s}$ and $\theta$ will be found by making a few approximations. To first order in $\phi_{s}$ and $\delta$, which are small when dissipation is weak, (27) becomes

$$
\phi_{s} \approx(\xi \cos \theta)^{-1}(\tan \theta \cot I-1) .
$$

Setting equal (28) and (29) with the above approximation gives the following constraint for $\theta$ :

$$
\begin{aligned}
\frac{\sin \theta}{\sin (\theta-I)}\left\{\left[\frac{M}{M_{p}}\left(\frac{R_{p}}{a}\right)^{5} \frac{2 k_{p}}{1+\sec ^{2} \theta}\right]^{2}+\left[\frac{1}{2 n t_{F p} \cos \theta}\right]^{2}\right\}^{1 / 2} & =\frac{C_{p}}{\mu h} k_{\star} \frac{M}{M_{\star}}\left(\frac{R_{\star}}{a}\right)^{5} \frac{\Omega_{\star}^{2}}{n} \frac{\sin \theta_{\star} \cos \theta_{\star}}{\sin I} .
\end{aligned}
$$

In this final equation, the right hand side is determined by parameters that are approximately constant over a planetary spin precession period. On the left hand side, the quantities that are uncertain are the planet's apsidal-motion constant and its tidal friction timescale. If the former is too big, or the latter is too small, no real solution for $\theta$ is possible, and there is no equilibrium corresponding to Cassini state 2. Tidal friction plays an important role here because with $t_{F p} \rightarrow \infty$, there is always a solution for $\theta$. Indeed, equation (6) is recovered in this limit5.

Starting from the values for $\Omega_{p}, \phi_{s}$, and $\theta$ given by equations (23), (30) and (31), a NewtonRaphson solver can efficiently find any solution to equations (23), (27), (28), and (29). Once $\theta$ is found, (23) can be used to find the planetary spin rate. Our derivation gives an especially physical understanding for why the spin cannot be truly synchronous: there needs to be a small component of $\dot{\Omega}_{\mathrm{p}}$ in the direction of the orbit normal so that the spin performs precession about the total angular momentum rather than the orbit normal.

\footnotetext{
${ }^{5}$ The spin precession constant $\alpha$ is related to the apsidal motion constant by

$$
\alpha=k_{p} \frac{\mu h}{C_{p}} \frac{M}{M_{p}}\left(\frac{R_{p}}{a}\right)^{5} \frac{\Omega_{p}}{n} .
$$
}




\section{Evolution of Cassini states}

First we will show the integration of equations (14) and (15) for an example that has an extremely tilted Cassini state 2. To find such a state we needed to take an extreme value for $k_{p}=5 \times 10^{-5}$, which corresponds to a polytrope $n=4.65$, instead of a more reasonable value such as $k_{p}=0.26$ for $n=1$. This choice lessens the planetary spin precession torque and dissipative torque relative to the rather weak orbital precession torque arising from the rotational bulge of the star. We also chose a quite large value of $Q_{p}^{\prime}=2.3 \times 10^{7}$. The planet started with a semimajor axis of $a=0.05 \mathrm{AU}$. The stellar spin began with a period of $10 \mathrm{~d}$ and made an angle $45^{\circ}$ with the orbit normal. The stellar apsidal motion constant was set to $k_{\star}=0.0144$, appropriate for an $n=3$ polytrope. The masses were $M_{\star}=M_{\odot}$ and $M_{p}=10^{-3} M_{\odot}$, radii $R_{\star}=1.0 R_{\odot}$ and $R_{p}=0.1 R_{\odot}$, and normalized moments of inertia $C_{\star} /\left(M_{\star} R_{\star}^{2}\right)=0.08$ and $C_{p} /\left(M_{p} R_{p}^{2}\right)=0.25$.

See Figure 4 for the evolution into Cassini state 2. At first the planetary spin axis librates with large amplitude, tracing curves of nearly constant Hamiltonian (Eq. 4). After about 20 Myr, dissipation has caused the system to settle to a state of quasi-equilibrium (for comparison, $\tau_{\perp}=4.3$ Myr). Panel (a) shows the evolution of the angles between the total angular momentum $\mathbf{J}$ and the orbit normal $\mathbf{n}$, between $\mathbf{J}$ and the stellar spin axis $\boldsymbol{\Omega}_{\star}$, and between $\mathbf{J}$ and the planetary spin axis $\boldsymbol{\Omega}_{\mathbf{p}}$. Panel (b) is a parametric plot (with time as the suppressed parameter) of the projection of the planetary spin vector $\hat{\Omega}_{\mathbf{p}}$ onto the $X Y$ plane of Figure 1. Panel (c) zooms in on the final state of panel (b), revealing that, in the presence of ongoing tidal dissipation, the equilibrium Cassini state 2 no longer lies on the $Y$-axis; it is shifted towards positive $X$ values by the tidal torque.

The long-term consequence of such a state will be shown next. We started with the same initial conditions, except set $Q_{p}^{\prime}=4.6 \times 10^{6}$ so that the evolution would proceed faster. An integration for 1.5 Gyr is shown in Figure 5. Panels (a) and (b) are the same as those of Figure 4. The timescale of damping into the Cassini state is a few Myr, smaller than the time axis resolution. Cassini state 2 now lies very far from the $Y$-axis, and evolves even farther from it. The state "breaks" when $Y=0$, then librates around, and damps into, Cassini state 1 . The physical cause is that the tidal torque (the last terms on the right hand sides of Eqs. 16] and 17, which are proportional to $a^{-9 / 2} Q^{\prime-1}$ ) overcomes the orbital precession torque (the first terms on the right hand sides of Eqs. [16] and 17, which are proportional to $a^{-3}$ ), as a decreases due to energy dissipation. Panel (c) shows the time dependence of the phase shift $\phi_{s}$ away from the $X$ axis (cf. Fig. 1). Most of the time is spent in the evolving equilibrium of state 2 , and the brief libration epochs that are prominent in panel (b) are not resolved in this plot. Panel (d) shows the semi-major axis evolution: dissipation in the planet converts orbital energy to heat energy. Panel (e) shows the evolution of the various angular momenta of the problem, projected along the total angular momentum axis. The angular momentum lost by the planetary orbit is absorbed by the stellar spin. However, in the absence of extremely strong dissipation in the star, the torque from the planet cannot cause the stellar spin period to change (Eq. 13). Panel (f) shows a parametric plot of the stellar spin vector broken into two components: those parallel to and perpendicular to the planet's orbit. It is clear that the star's spin absorbs the angular momentum of the planetary orbit by reorienting, not by 
changing magnitude.

The non-zero value of $\phi_{s}$ causes the stellar spin to come into alignment with the planet's orbit, without any dissipation in the star, by the following mechanism. The spin angular momentum of the planet is not coplanar with the other angular momenta, which causes the total angular momentum $\mathbf{J}$ to lie slightly outside of the plane defined by the dominant angular momenta $\mathbf{L}$ and $\mathbf{S}_{\star}$. The evolution equations cause $\mathbf{S}_{\star}$ to precess about $\mathbf{L}$ (there is no spin-spin coupling), but this non-coplanarity directs a small component of that torque towards J. Similarly, the motion of the orbital angular momentum is not quite the pure precession of equation (28) because the small coupling of the orbit to the rotational bulge of the planet $\left(\mathcal{X}_{p}\right.$ and $\mathcal{Y}_{p}$ of Eq. 14) causes a secular motion of the orbit normal towards $\mathbf{J}$. Therefore the stellar spin and planet orbit come into alignment with the total angular momentum, and thus with each other. Recall that the ingredients for this alignment are a close planet whose spin is trapped in Cassini state 2, whose tidal dissipation causes that state to shift in phase. In the example we have given, a significant spinorbit misalignment persists because the planet's spin left Cassini state 2 before all of the available angular momentum from the star was tapped.

In integrations like that of Figure 4 (in which $\phi_{s}=0.1106$ ), the first-order estimate of $\phi_{s}$ (Eq. 30) is quantitatively quite good, and its fidelity for larger values can be seen in Figure 5(c). It is remarkable that the total amount of orbital energy dissipated is many orders of magnitude beyond that which is stored in the planet's spin; it is approximately the binding energy of the planet! The average dissipation rate indicated by the orbital migration of Figure 5 (d) is $\dot{E} \approx 6.2 \times 10^{43} \mathrm{erg} \mathrm{s}^{-1}$, enough to inflate the planet's radius (during the first $1.1 \mathrm{Gyr}$ after Cassini state 2 is established). However, we achieved this result by adopting an apsidal moment constant almost four orders of magnitude smaller than Jupiter's, corresponding to a very strongly centrally concentrated body, in order to reduce the spin precession rate and dissipative tidal torque. Such a value for $k_{p}$ would be much more strongly discrepant with theory than the inflated radius itself.

To seek the range of structural parameters that allow Cassini state 2 to be an equilibrium, we may use $\phi_{s}=1$ in equation (30) to approximately indicate when Cassini state 2 will break due to dissipation in the planet. This first order estimate actually is serendipitously rather precise: recognizing from Figure 5 that Cassini state 2 actually breaks when $\phi_{s}=\pi / 2$, we may substitute this value into equation (27). The result is that the right hand side of (30) equals 1 . That is, the full phase shift equation gives $\phi_{s}=\pi / 2$ when the first order estimate gives $\phi_{s}=1$, which can be seen in Figure [5(c).

What is the maximum energy dissipation rate a planet may experience while in Cassini state 2, driven by stellar bulge orbital precession? A closed equation for $\dot{E}_{\text {max }}$ may be written by making a few approximations (all of which, we have verified, are well-justified). For realistic planetary structure, $k_{p}=0.26$, which requires $\theta \approx \pi / 2$. In the evaluations below, we will also use $k_{\star}=0.0144$, $R_{\star}=R_{\odot}, M_{\star}=M_{\odot}, C_{\star} /\left(M_{\star} R_{\star}^{2}\right)=0.08, \Omega_{\star}=2 \pi /(20 \mathrm{~d}), \theta_{\star}=0.1, R_{p}=R_{J}, M_{p}=M_{J}$, $C_{p} /\left(M_{p} R_{p}^{2}\right)=0.25$, and $n=2 \pi /(4 \mathrm{~d})$. Expanding $g$ (Eq. 29) to first order in $\pi / 2-\theta$, while 
neglecting the dissipative term and setting $\sin \beta \rightarrow 1$, then setting the resulting expression equal to equation (28) gives

$$
\theta \approx \pi / 2-\left(\frac{k_{\star} R_{\star}^{5}}{2 k_{p} R_{p}^{5}} \frac{a C_{p} \Omega_{\star}^{2}}{G M_{\star}^{2}} \frac{\sin \theta_{\star} \cos \theta_{\star}}{\sin I}\right)^{1 / 2} .
$$

Second, the solution will have an extreme value for $\phi_{s}$, which we may identify by setting the first order estimate (Eq. 30) to unity. This allows us to identify the $Q_{p}$ parameter for such a maximally dissipating planet:

$$
Q_{p, \min }=\frac{3}{4} \frac{M_{\star}}{M} \frac{(\pi / 2-\theta)^{-3}}{\tan I} .
$$

For the nominal values above, this means $Q_{p} \geq 8 \times 10^{8}$ for Cassini state 2 to be an equilibrium, where equality holds for a state barely in equilibrium, with the planet dissipating at the maximal value. That the population of hot Jupiters is inferred to have $Q_{p} \approx 10^{6}(\sqrt[3.3]{3})$ implies that a planet must be unusually non-dissipative to remain in state 2. Finally, the energy dissipation $\dot{E}_{\text {max }}=G^{2} M_{\star} M_{p} M h^{-3} \dot{h}$ may be written

$$
\begin{aligned}
\dot{E}_{\max } & =(9 / 4) Q^{\prime-1} G M_{\star}^{2} R_{p}^{5} a^{-6} n \\
& =\frac{2^{1 / 2} k_{\star}^{3 / 2}}{k_{p}^{1 / 2}} \frac{M^{3 / 2}}{M_{\star}^{2}} \frac{R_{\star}^{15 / 2}}{R_{p}^{5 / 2}} \frac{C_{p}^{3 / 2} \Omega_{\star}^{3}}{a^{6}}\left(\frac{\sin ^{3} \theta_{\star} \cos ^{3} \theta_{\star}}{\sin I \cos ^{2} I}\right)^{1 / 2},
\end{aligned}
$$

which evaluates to $\dot{E}_{\max } \approx 5 \times 10^{23} \mathrm{erg} \mathrm{s}^{-1}$ given the above values for the parameters. The maximum possible rate of heating due to orbital precession-stabilized obliquity tides is comparable to the luminosity of gravitational contraction. That is, if a certain transiting planet is in Cassini state 2, then tidal dissipation is not strong enough to inflate that planet's radius. Equation (34) has a strong dependence on semimajor axis, but even a Jupiter mass planet at its Roche limit cannot dissipate a structurally significant power via Cassini state 2, even if it somehow managed to have $Q_{p} \gtrsim Q_{p, \min }$.

Another way to assess stability is to follow Levrard et al. (2007) in requiring that the dissipative torque does not destroy $\theta$ stability (that a real solution exists for $\theta$ in Eq. 31). For the above parameters, this gives $\dot{E}_{\max } \approx 6 \times 10^{24} \mathrm{erg} \mathrm{s}^{-1}$, a factor of $\sim 10$ less restrictive than our criterion that the first order estimate of $\phi_{s}$ is less than 1 . We interpret this to mean that Cassini equilibrium is generally lost in the $\phi$ direction (as in Fig. [5] ), not the $\theta$ direction.

\section{Discussion and Conclusions}

We have calculated the stability and evolution of Cassini state 2 with large obliquity $\theta$ in the presence of tidal dissipation. We have verified that in principle, a spin state can couple the orbit to internal energy such that many times the energy of the spin is dissipated. However, it cannot dissipate the energy rapid enough for that heat to make a structural difference. Here we apply the concepts we have developed to the archetypal inflated planet, HD 209458b, and find that neither the stellar bulge nor a second planet can be responsible for a planet-inflating Cassini state. 
According to Bodenheimer et al. (2003), if the large radius of HD 209458b is due to internal heating, the inferred heating rate $\dot{E}$ is at least $4 \times 10^{26} \mathrm{erg} \mathrm{s}^{-1}$. Can this $\dot{E}$ be continually supplied by obliquity tides over the age of the planet, without the Cassini state breaking?

In 95 we evaluated the hypothesis that the stellar bulge causes the orbital precession, finding that the tidal torque causes Cassini state 2 to fall out of equilibrium. When numbers appropriate to the HD 209458 system - including a plausible planetary apsidal-motion constant - are used in equation (34), the maximum possible heating rate is $3 \times 10^{24} \mathrm{erg} \mathrm{s}^{-1}$, a factor of $\sim 100$ too small to explain the inflated radius.

Independent of this dissipative torque constraint, we note that the usable angular momentum in the spin, as the star comes into alignment, is quite small given the observationally indicated stellar obliquity. The angular momentum that the stellar spin can absorb from the orbit before complete alignment, using equation (8) with $L_{2} \rightarrow S_{\star}$ and $i_{\text {rel, } \mathrm{i}} \rightarrow \theta_{\star}$, is

$$
\Delta L=L_{f}-L=\left(L^{2}+S_{\star}^{2}+2 L S_{\star} \cos \theta_{\star}\right)^{1 / 2}-S_{\star}-L .
$$

So for HD 209458, taking $\theta_{\star}=0.1$, we have $\Delta L \approx-2.1 \times 10^{46} \mathrm{~g} \mathrm{~cm}^{2} \mathrm{~s}^{-1}$. The quoted $\dot{E}$ implies $\dot{L} \approx-1.9 \times 10^{31} \mathrm{~g} \mathrm{~cm}^{2} \mathrm{~s}^{-2}$. Then, if the angular momentum is lost at a steady rate, the system will become coplanar and the high obliquity state will end in only $\sim 35 \mathrm{Myr}$, which is $\lesssim 1 \%$ of the system's age (5 Gyr; Cody \& Sasselov 2002). Under this hypothesis we are seeing the system at a very special time, just before it becomes coplanar.

Alternatively, a second planet, rather than stellar obliquity, may be responsible for the orbital precession that has HD 209458b trapped in Cassini state 2 (see 3.2 ). Here we compile the constraints and find that this hypothesis is also ruled out (Fig. 6). Briefly, a putative second planet must be massive and distant enough to absorb the angular momentum of the dissipating planet, yet close enough so that its torque can compete with the dissipative torque, and these requirements would violate the radial velocity constraints. Each of these constraints is explained next.

How much angular momentum must be transfered to the second planet? If we happen to be observing the system near the end of Cassini state 2, such that it is nearly coplanar now, the final angular momentum in the orbit of HD 209458b is $L_{f}=L_{\text {now }}=1.26 \times 10^{49} \mathrm{~g} \mathrm{~cm}^{2} \mathrm{~s}^{-1}$. We may combine the current inferred rate of orbital evolution with the expected scaling of $\dot{L}$ with $L$ to find its primordial value (3.2). Assuming Cassini state 2 was established at the inception of the system, the planet's primordial angular momentum was $L_{i} \approx 1.41 \times 10^{49} \mathrm{~g} \mathrm{~cm}^{2} \mathrm{~s}^{-1}$. Thus the second planet must have enough non-aligned angular momentum such that $\Delta L_{2}=1.5 \times 10^{48} \mathrm{~g} \mathrm{~cm}^{2} \mathrm{~s}^{-1}$ is absorbed before coplanarity is established. If we had assumed that the Cassini state was only midway through its evolution, this requirement would be even more strict.

Using these values, we have that the primordial value for $i_{\text {rel,i }}$ must have been greater than $i_{\text {crit }}=27^{\circ}$. This value is considerably larger than relative inclinations of planets in our solar system, but it may be envisioned that planet-planet scattering, followed by planet-star tidal dissipation, was the cause of HD 209458b's current small semi-major axis, after which $i_{\text {rel }}$ may plausibly be 
that large (Marzari \& Weidenschilling 2002). On the other hand, for very large initial inclinations, eccentricity oscillations by the Kozai mechanism plus tidal friction will reduce $i_{\text {rel }}$ to $\sim 40^{\circ}$ before obliquity tides start to dominate dissipation, so it is reasonable to consider only initial values of $i_{\text {rel,i }}$ less than $40^{\circ}$.

For each assumed primordial relative inclination, the second planet must have at least the orbital angular momentum $L_{2}$ of equation (9) so that the two orbits are not yet coplanar. This lower limit gives a lower limit to the mass of the putative planet as a function of $a_{2}\left(1-e_{2}^{2}\right)$, which we plot in Figure 6 for two values of $i_{\text {rel,i }}$.

At large orbital separation, the torque of the second planet will not be able to compete with the dissipative torques, and by analogy with the stellar oblateness analysis, Cassini state 2 will not be an equilibrium. Here equation (30) still applies, but $\theta$ must be determined by setting the planet's spin precession rate $g$ of equation (29) equal to

$$
g_{2}=\frac{3}{4} \frac{G^{1 / 2} M_{2}}{M^{1 / 2}} \frac{a^{3 / 2} \cos i_{\mathrm{rel}, \mathrm{now}}}{a_{2}^{3}\left(1-e_{2}^{2}\right)^{3 / 2}}
$$

which is the orbital precession rate due to the second planet, whose mass is $M_{2}$, semimajor axis $a_{2}$, eccentricity $e_{2}$, and current relative inclination $i_{\text {rel,now }}$. Here $g_{2}$ is calculated by secular theory, and any enhancement to this rate by the effects of mean motion resonance will only be temporary, since $a$ is evolving due to tidal dissipation. To find the critical parameters of a second planet which barely compete with the dissipation torque, we may set $\phi_{s}=1$ in equation (30), and similar approximations as those that led to equations (32)-(34) give the constraint

$$
\frac{M_{2}}{a_{2}^{3}\left(1-e_{2}^{2}\right)^{3 / 2}}>\frac{2 \times 4^{1 / 3}}{3} \dot{E}^{2 / 3} \frac{M_{\star}^{1 / 3}}{G} \frac{k_{p}^{1 / 3}}{C_{p}} \frac{R_{p}^{5 / 3}}{a} \frac{\cot ^{2 / 3} I}{\cos i_{\text {rel,now }}} .
$$

If the exterior planet's orbit dominates the angular momentum, $i_{\text {rel,now }} \approx I$; the minimum value of $\cot ^{2 / 3} I / \cos I$ occurs at $I=\tan ^{-1} \sqrt{2} \approx 54.7^{\circ}$ and has the value $3^{1 / 2} / 2^{1 / 3} \approx 1.375$. This angle is bigger than the critical angle for Kozai eccentricity oscillations, so taking 1.375 for the multiplicative factor depending on the inclinations is quite conservative. Then, evaluating the other terms with the values cited for $\mathrm{HD} 209458 \mathrm{~b}$ gives $M_{2} a_{2}^{-3}\left(1-e_{2}^{2}\right)^{-3 / 2}>3.74 M_{J} \mathrm{AU}^{-3}$. This constraint is labeled "dissipative torque" on Figure 6, It scales with $e_{2}$ differently than the angular momentum constraint, but the limit shown is true for all eccentricities.

The parameter space of possible second planets is also constrained by the lack of a secondary signal in the radial velocity data, giving a mass upper limit plotted in Figure6. No part of parameter space remains, so the second planet mechanism for causing the inflation of HD 209458b via Cassini state 2 is ruled out.

We have ruled out both the stellar rotational bulge and a second planet as drivers of a high obliquity Cassini state for HD 209458b, if it is presumed that obliquity tides are inflating its radius. We have focused on this planet as the observational material far surpasses that of other planets. 
However, we expect that the hypothesis also fails for the other anomalously large planets HATP-1b and WASP-1b. Likewise, planetary systems discovered in the future will need very peculiar parameters if obliquity tides are to be an important heating source.

\section{Acknowledgments}

DF was supported by a NASA Origins of Solar Systems Program grant to S. Tremaine, and EJ by NAG5-11664 to JG. We thank Joshua Winn for detailed and helpful comments on a draft of this paper.

\section{REFERENCES}

Arras, P., \& Bildsten, L. 2006, ApJ, 650, 394

Bakos, G. A., et al. 2006, preprint (astro-ph/0609369)

Baraffe, I., Chabrier, G., Barman, T. S., Allard, F., \& Hauschildt, P. H. 2003, A\&A, 402, 701

Barman, T. S., Hauschildt, P. H., \& Allard, F. 2005, ApJ, 632, 1132

Beletskii, V. V. 1972, Celestial Mechanics, 6, 356

Bodenheimer, P., Laughlin, G., \& Lin, D. N. C. 2003, ApJ, 592, 555

Bodenheimer, P., Lin, D. N. C., \& Mardling, R. A. 2001, ApJ, 548, 466

Bouchy, F., et al. 2005, A\&A, 444, L15

Bouchy, F., Pont, F., Santos, N. C., Melo, C., Mayor, M., Queloz, D., \& Udry, S. 2004, A\&A, 421, L13

Burkert, A., Lin, D. N. C., Bodenheimer, P. H., Jones, C. A., \& Yorke, H. W. 2005, ApJ, 618, 512

Burrows, A., Hubeny, I., Budaj, J., \& Hubbard, W. B. 2006a, preprint (astro-ph/0612703)

Burrows, A., Hubeny, I., \& Sudarsky, D. 2005, ApJ, 625, L135

Burrows, A., Sudarsky, D., \& Hubbard, W. B. 2003, ApJ, 594, 545

Burrows, A., Sudarsky, D., \& Hubeny, I. 2006b, ApJ, 650, 1140

Charbonneau, D., et al. 2005, ApJ, 626, 523

Cody, A. M., \& Sasselov, D. D. 2002, ApJ, 569, 451

Colombo, G. 1966, AJ, 71, 891 
Cooper, C. S., \& Showman, A. P. 2005, ApJ, 629, L45

Deming, D., Harrington, J., Seager, S., \& Richardson, L. J. 2006, ApJ, 644, 560

Deming, D., Seager, S., Richardson, L. J., \& Harrington, J. 2005, Nature, 434, 740

Eggleton, P. P., Kiseleva, L. G., \& Hut, P. 1998, ApJ, 499, 853

Eggleton, P. P., \& Kiseleva-Eggleton, L. 2001, ApJ, 562, 1012

Ford, E. B., Kozinsky, B., \& Rasio, F. A. 2000, ApJ, 535, 385

Fortney, J. J., Marley, M. S., \& Barnes, J. W. 2006a, preprint (astro-ph/0612671)

Fortney, J. J., Marley, M. S., Lodders, K., Saumon, D., \& Freedman, R. 2005, ApJ, 627, L69

Fortney, J. J., Saumon, D., Marley, M. S., Lodders, K., \& Freedman, R. S. 2006b, ApJ, 642, 495

Goldman, I., \& Mazeh, T. 1991, ApJ, 376, 260

Goldreich, P., \& Nicholson, P. D. 1977, Icarus, 30, 301

Goldreich, P., \& Soter, S. 1966, Icarus, 5, 375

Grillmair, C. J., Charbonneau, D., Burrows, A., Armus, L., Stauffer, J., Meadows, V., van Cleve, J., \& Levine, D. 2007, preprint (astro-ph/0702494)

Guillot, T. 2005, Annual Review of Earth and Planetary Sciences, 33, 493

Guillot, T., \& Showman, A. P. 2002, A\&A, 385, 156

Harrington, J., Hansen, B. M., Luszcz, S. H., Seager, S., Deming, D., Menou, K., Cho, J. Y.-K., \& Richardson, L. J. 2006, Science, 314, 623

Henrard, J., \& Murigande, C. 1987, Celestial Mechanics, 40, 345

Iro, N., Bézard, B., \& Guillot, T. 2005, A\&A, 436, 719

Knutson, H. A., Charbonneau, D., Noyes, R. W., Brown, T. M., \& Gilliland, R. L. 2007, ApJ, 655, 564

Laughlin, G., Marcy, G. W., Vogt, S. S., Fischer, D. A., \& Butler, R. P. 2005a, ApJ, 629, L121

Laughlin, G., Wolf, A., Vanmunster, T., Bodenheimer, P., Fischer, D., Marcy, G., Butler, P., \& Vogt, S. 2005b, ApJ, 621, 1072

Levrard, B., Correia, A. C. M., Chabrier, G., Baraffe, I., Selsis, F., \& Laskar, J. 2007, A\&A, 462, L5 
Maness, H. L., Marcy, G. W., Ford, E. B., Hauschildt, P. H., Shreve, A. T., Basri, G. B., Butler, R. P., \& Vogt, S. S. 2007, PASP, 119, 90

Marzari, F., \& Weidenschilling, S. J. 2002, Icarus, 156, 570

Mathieu, R. D., Duquennoy, A., Latham, D. W., Mayor, M., Mermilliod, T., \& Mazeh, J. C. 1992, in Binaries as Tracers of Stellar Formation (Cambridge, England: Cambridge University Press), ed. A. Duquennoy \& M. Mayor, 278

Mathieu, R. D., Meibom, S., \& Dolan, C. J. 2004, ApJ, 602, L121

McCullough, P. R., et al. 2006, ApJ, 648, 1228

Melo, C., Santos, N. C., Pont, F., Guillot, T., Israelian, G., Mayor, M., Queloz, D., \& Udry, S. 2006, A\&A, 460, 251

Miralda-Escudé, J. 2002, ApJ, 564, 1019

Murray, C. D., \& Dermott, S. F. 1999, Solar system dynamics (Cambridge University Press)

O’Donovan, F. T., et al. 2006, ApJ, 651, L61

Ogilvie, G. I., \& Lin, D. N. C. 2004, ApJ, 610, 477

—. 2007, preprint (astro-ph/0702492)

Peale, S. J. 1969, AJ, 74, 483

-. 1974, AJ, 79, 722

Pont, F., Bouchy, F., Queloz, D., Santos, N. C., Melo, C., Mayor, M., \& Udry, S. 2004, A\&A, 426, L15

Queloz, D., Eggenberger, A., Mayor, M., Perrier, C., Beuzit, J. L., Naef, D., Sivan, J. P., \& Udry, S. 2000, A\&A, 359, L13

Richardson, L. J., Deming, D., Horning, K., Seager, S., \& Harrington, J. 2007, preprint (astro$\mathrm{ph} / 0702507)$

Sato, B., et al. 2005, ApJ, 633, 465

Seager, S., Richardson, L. J., Hansen, B. M. S., Menou, K., Cho, J. Y.-K., \& Deming, D. 2005, ApJ, 632, 1122

Showman, A. P., \& Guillot, T. 2002, A\&A, 385, 166

Shporer, A., Tamuz, O., Zucker, S., \& Mazeh, T. 2006, preprint (astro-ph/0610556)

Skumanich, A. 1972, ApJ, 171, 565 
Swain, M. R., Bouwman, J., Akeson, R., Lawler, S., \& Beichman, C. 2007, preprint (astro$\mathrm{ph} / 0702593)$

Valenti, J. A., \& Fischer, D. A. 2005, ApJS, 159, 141

Ward, W. R. 1975, AJ, 80, 64

Ward, W. R., Burns, J. A., \& Toon, O. B. 1979, J. Geophys. Res., 84, 243

Ward, W. R., \& Canup, R. M. 2006, ApJ, 640, L91

Ward, W. R., \& Hamilton, D. P. 2004, AJ, 128, 2501

Winn, J. N. 2006, preprint (astro-ph/0612744)

Winn, J. N., et al. 2005, ApJ, 631, 1215

-. 2006a, ApJ, 653, L69

—. 2006b, preprint (astro-ph/0612224)

Winn, J. N., \& Holman, M. J. 2005, ApJ, 628, L159

Wu, Y. 2005, ApJ, 635, 688

Zahn, J. P. 1966, Annales d'Astrophysique, 29, 489

-. 1970, A\&A, 4, 452

Zahn, J.-P., \& Bouchet, L. 1989, A\&A, 223, 112 


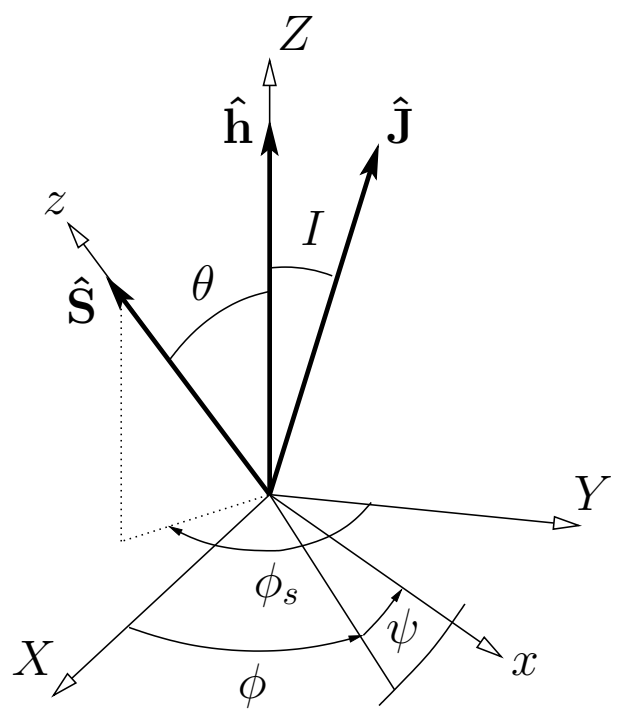

Fig. 1.- The $X Y Z$ coordinate system precesses with angular frequency $g$ about the direction of the total angular momentum $\hat{\mathbf{J}}$. The orbital plane, with normal $\hat{\mathbf{h}}$ along the $Z$-axis, is inclined to the invariable plane by angle $I$ with the ascending node in the $X$ direction ( $\hat{\mathbf{J}}$ lies in the $Y Z$ plane). The $x y z$ axes are the principal axes of the planet, with the unit spin vector $\hat{\mathbf{S}}$ along the axis of symmetry. $\phi, \psi$, and the obliquity $\theta$ are Euler angles. The phase angle $\phi_{s} \equiv \pi-\phi$. Nondissipative Cassini states have $\hat{\mathbf{S}}$ in the $Y Z$ plane, with $\phi_{s}=\pi(0)$ for state 1 (2); but dissipation causes $0<\phi_{s} \lesssim \pi / 2$ in state 2 . 

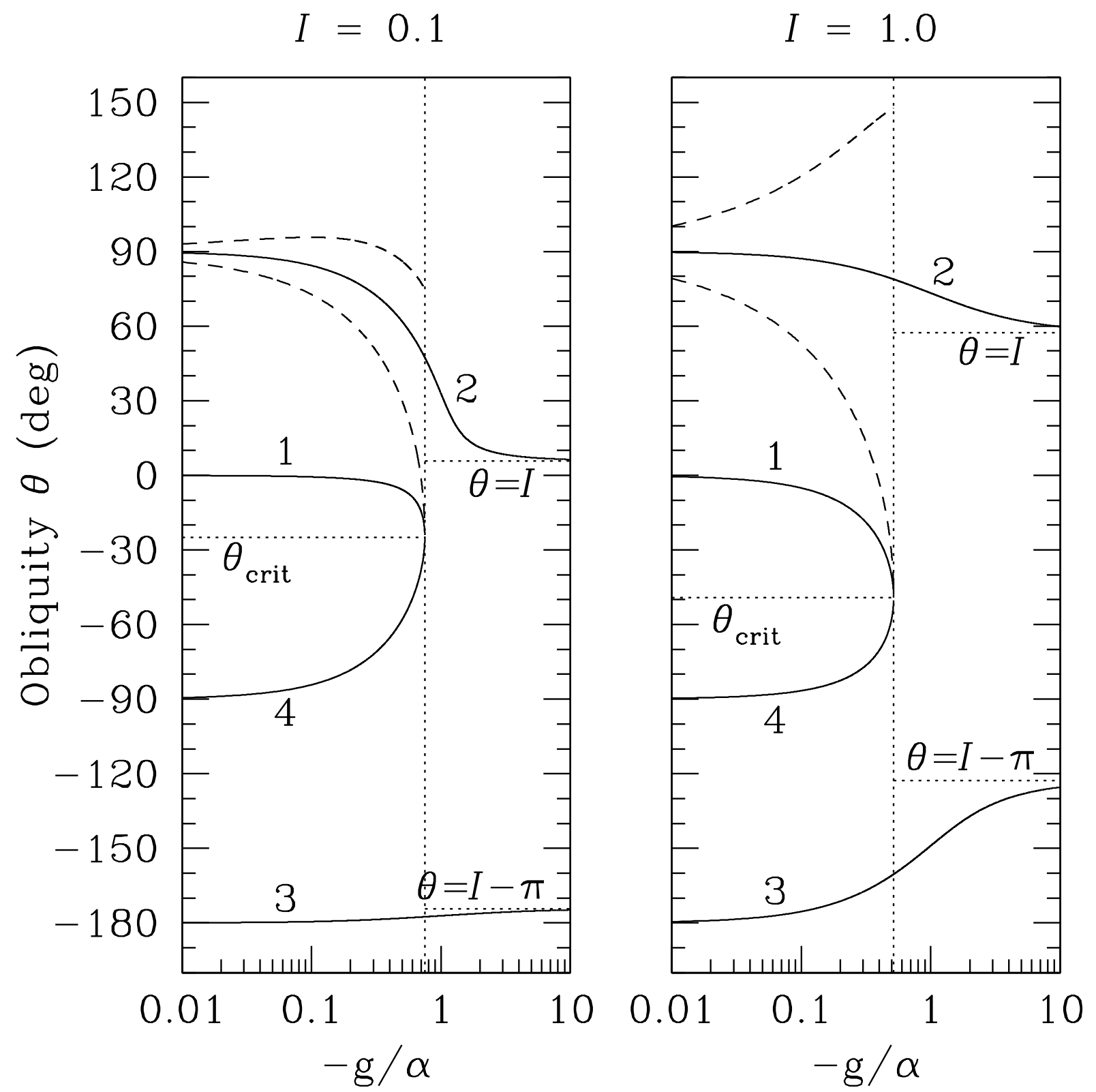

Fig. 2.- Obliquities of the four Cassini states as functions of $-g / \alpha$. Solid numbered curves are the solutions of equation (6) for $I=0.1$ (left panel) and $I=1.0$ (right panel). Cassini states lie in the $Y Z$ plane of Figure 1, by convention $\theta$ is positive when $\phi_{s}=0$ and negative when $\phi_{s}=\pi$. The separatrix intersects the $Y Z$ plane at state 4 , and at the angles indicated by the dashed curves. States 1 and 4 merge and vanish at the critical value $(-g / \alpha)_{\text {crit }}=\left(\sin ^{2 / 3} I+\cos ^{2 / 3} I\right)^{-3 / 2}$ (dotted vertical line). Three values of $\theta$ are indicated by dotted horizontal lines. The largest absolute obliquity attained by state 1 is $\theta_{\text {crit }}=\tan ^{-1}\left(-\tan ^{1 / 3} I\right)$. States 2 and 3 approach $\theta=I$ and $\theta=I-\pi$, respectively, when $|g / \alpha| \gg 1$. 


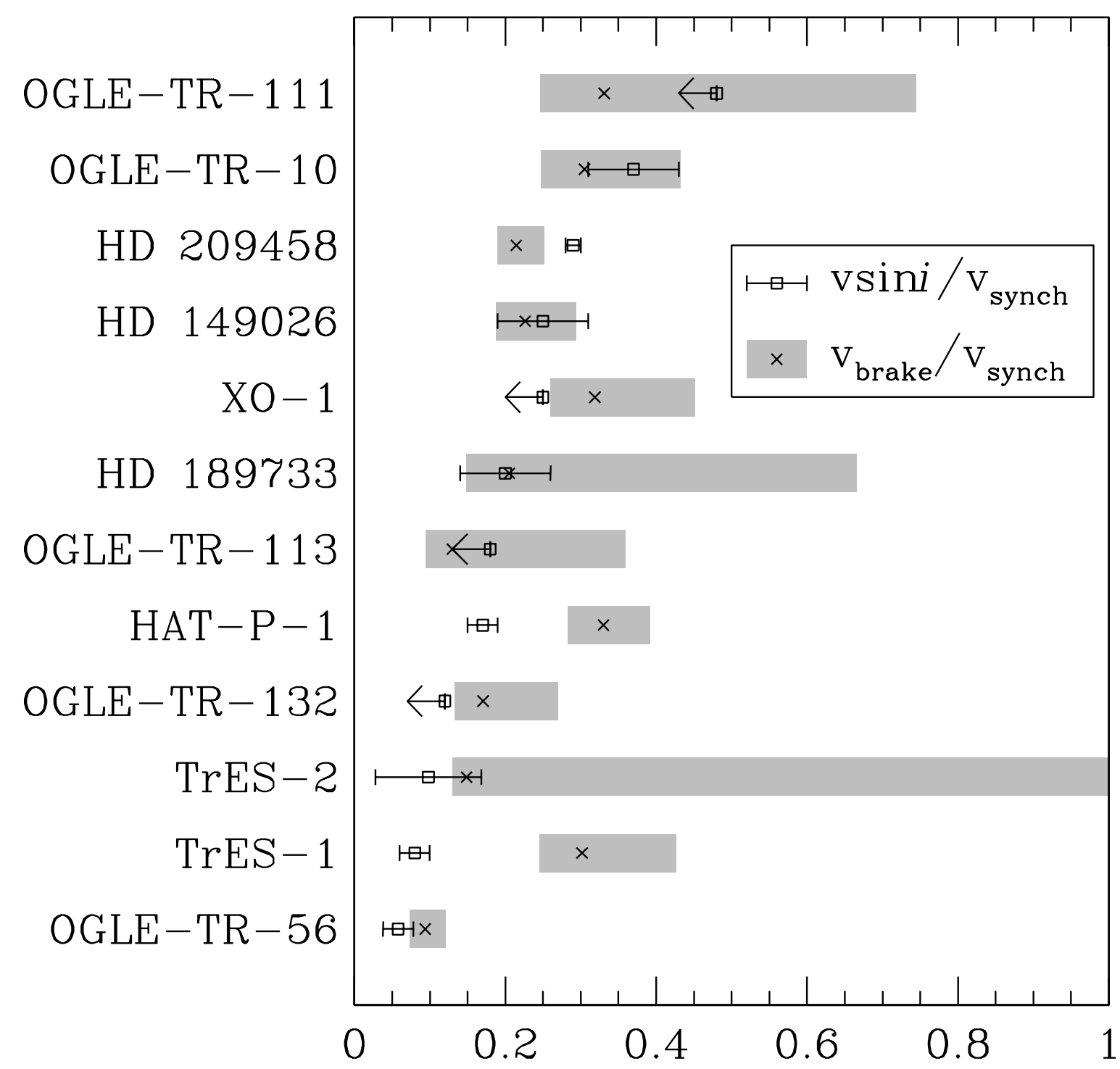

Fig. 3.- Measured stellar rotational velocity $(v \sin i)_{\star}$ relative to synchronous rotation $v_{\mathrm{synch}}=$ $n R_{\star}$ (squares). Stars bearing transiting planets are listed top to bottom in order of decreasing $v \sin i / v_{\text {synch. }}$. Error bars indicate observational uncertainty in $(v \sin i)_{\star}$ and $R_{\star}$. We compute

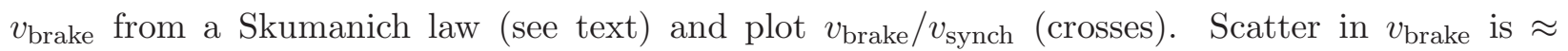
$10 \%$. Gray boxes give a range for $v_{\text {brake }} / v_{\text {synch }}$ corresponding to the $1 \sigma$ uncertainty in stellar age (systematic uncertainties are not accounted for). Probably all stars are rotating subsynchronously, since $v \sin i \approx v$ for a transiting planet whose orbit is nearly coplanar with the equator of its host star. Only HD 209458 is rotating (marginally) more rapidly than would be predicted by magnetic braking alone. Data are taken from Burrows et al. (2006a) and references therein, except for the values of $(v \sin i)_{\star}$, which are taken from the following sources, top to bottom: Pont et al. (2004), Melo et al. (2006), Winn et al. (2005), Sato et al. (2005), McCullough et al. (2006), Bouchy et al. (2005), Bouchy et al. (2004), Bakos et al. (2006), Bouchy et al. (2004), O'Donovan et al. (2006), Laughlin et al. (2005b), and Melo et al. (2006). 

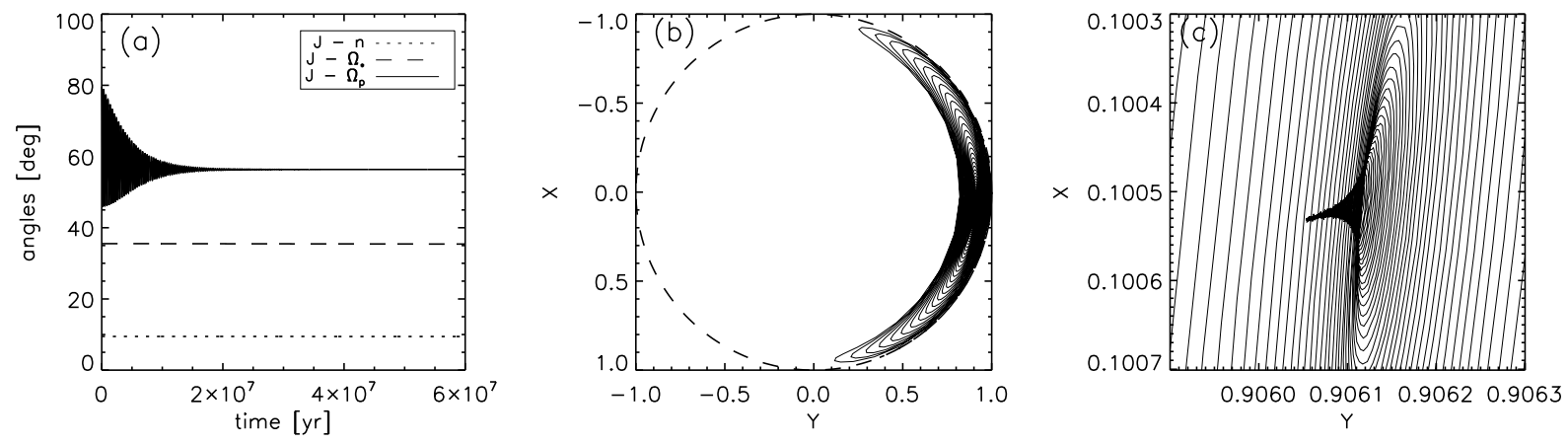

Fig. 4.- Settling into Cassini state 2. (a) The angles between $\mathbf{J}$ and the other vectors as a function of time. (b) The motion of $\hat{\Omega}_{\mathbf{p}}$ on the unit sphere, in the coordinate system of Figure 1. (c) After the equilibrium state is reached, the system slowly evolves (see Fig. [5). Notice that the state does not have equilibrium value $\phi=\pi$, as expected from the analysis with no dissipation. In this case there is a phase shift away from the $\mathrm{Y}$ axis of $\phi_{s}=0.1105$. We have used an exceptionally low value of the apsidal motion constant $k_{p}=5 \times 10^{-5}$ for this integration (see text). 

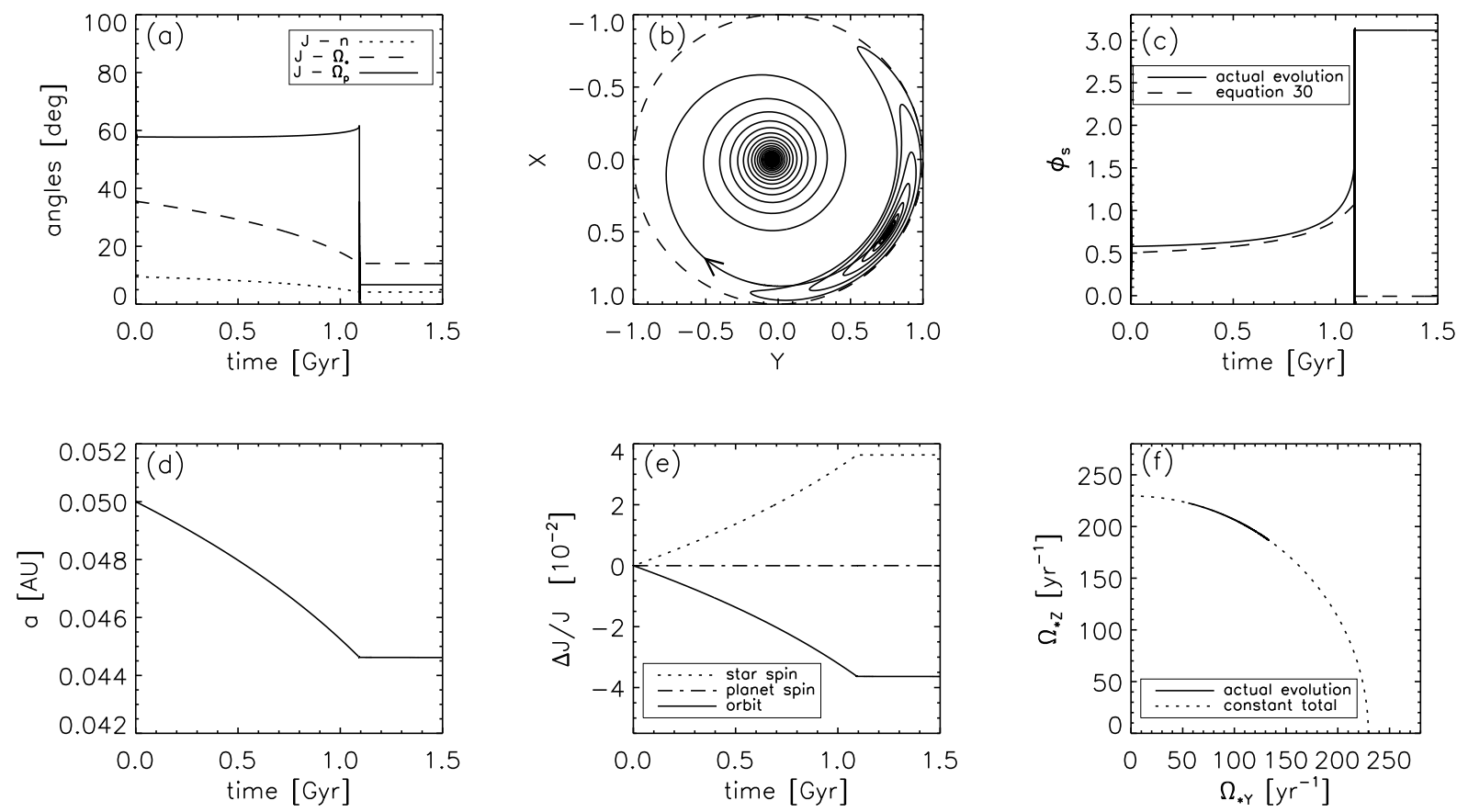

Fig. 5.- The long term evolution of Cassini state 2. (a) The angles between $\mathbf{J}$ and the other vectors as a function of time. (b) The motion of $\hat{\Omega}_{\mathbf{p}}$ on the unit sphere, in the coordinate system of Figure 1. This longer integration shows that after damping into Cassini state 2, as in Figure 4(b), the planetary spin vector evolves to negative $\mathrm{Y}$, after which equilibrium is lost it librates around, and damps into, Cassini state 1. (c) The evolution of the phase shift angle $\phi_{s} \equiv \pi-\phi$. Most of the time in the parametric plot of panel (b) is spent in the evolving state 2; libration epochs are too short to be resolved on this plot. The dashed line is the first-order estimate of equation (30). (d) The obliquity tides cause the semi-major axis to shrink. After damping to Cassini state 1, no appreciable energy is lost. (e) The changes in the projections of the three angular momenta along the $\hat{\mathbf{J}}$ axis, as a function of time. The angular momentum lost by the planet as it migrates in is absorbed by the stellar spin. (f) The stellar spin absorbs the angular momentum of the planetary orbit by reorienting toward alignment. The dashed line is the set of points with equal magnitude of $\Omega_{\star}$, showing that the only change to the stellar spin is its direction. We have used an exceptionally low value of the apsidal motion constant $k_{p}=5 \times 10^{-5}$ for this integration (see text). 


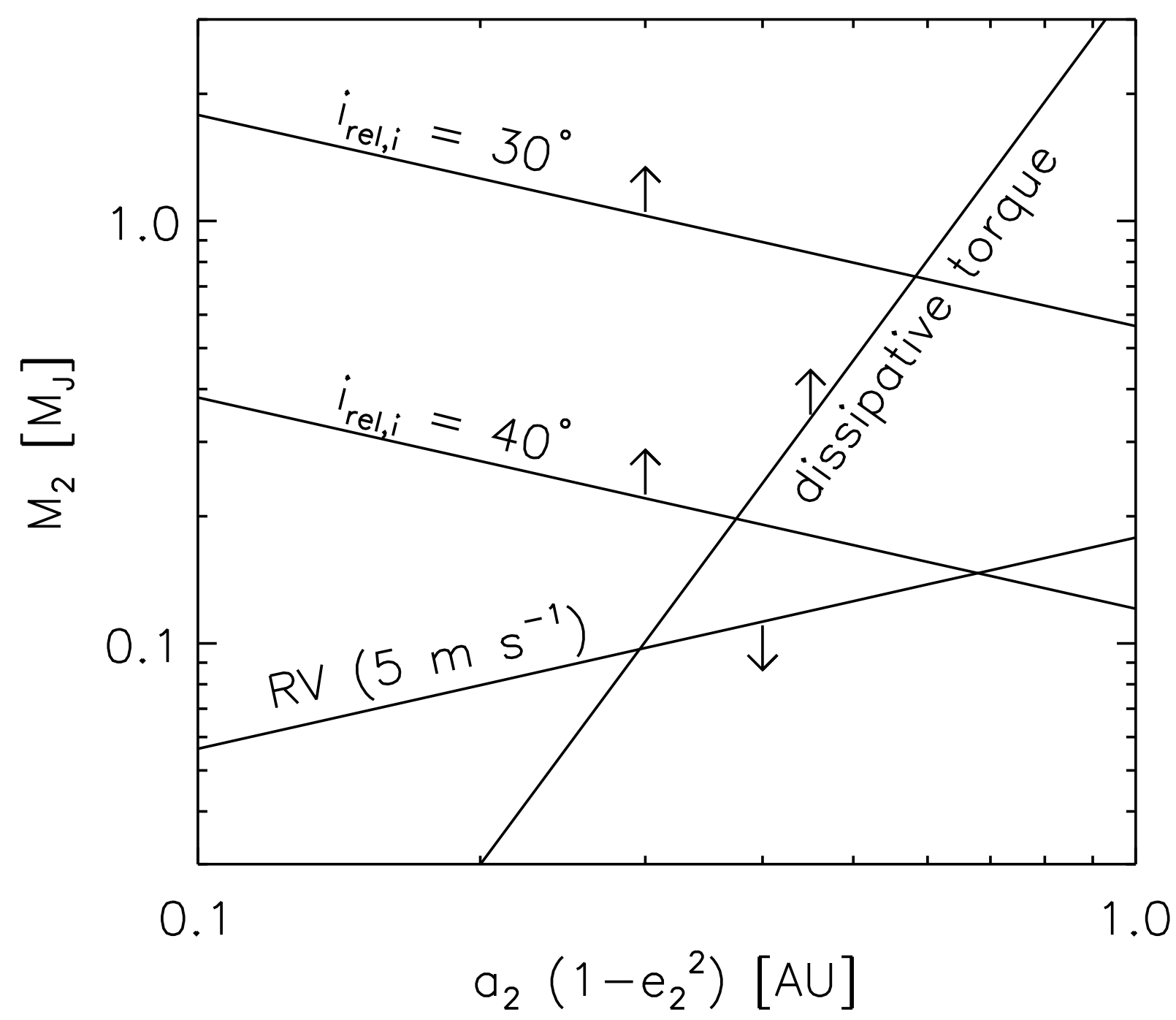

Fig. 6. - The limits of mass $M_{2}$ and orbital elements $a_{2}\left(1-e_{2}^{2}\right)$ of a second planet for the hypothesis that it is responsible for the inflation of HD 209458b via a Cassini resonance. Here $i_{\text {rel,i }}$ is the initial relative inclination between the two planets, and the bounds so labeled reflect the tendency of the second planet's orbit to align as it absorbs the angular momentum shed by the orbit of HD 209458b ( $L_{2}$ must be greater than that of eq. 9). Values of $i_{\text {rel,i }}>40^{\circ}$ are unlikely as the second planet would force Kozai eccentricity oscillations in the transiting planet. The curve labeled "dissipative torque" is the lower limit of mass needed to overcome the tidal dissipation that tends to break Cassini state 2 (eq. 37). The radial velocity dataset also constrains possible second planets; absence of a secondary signal of amplitude $K=5 \mathrm{~m} \mathrm{~s}^{-1}$ puts an upper limit on mass at each orbital distance. The arrows point to the portion of parameter space allowed by each particular constraint. Apparently all of parameter space is ruled out: tides due to a high obliquity state, stabilized by the orbital precession induced by a second planet, cannot significantly inflate HD 209458b. See $\sqrt[6]{6}$ for derivation and details. 